\title{
Structure activity relationship of phenolic acid inhibitors of $\alpha$-synuclein fibril formation and toxicity
}

\author{
Mustafa T. Ardah ${ }^{1}$, Katerina E. Paleologou ${ }^{2}$, Guohua Lv ${ }^{3}$, Salema B. Abul Khair ${ }^{1}$, Abdulla S. Kazim ${ }^{1}$, \\ Saeed T. Minhas ${ }^{4}$, Taleb H. Al-Tel ${ }^{5}$, Abdulmonem A. Al-Hayani ${ }^{6}$, Mohammed E. Haque $^{1}$, David Eliezer ${ }^{3}$ \\ and Omar M. A. El-Agnaf ${ }^{1,7 *}$
}

${ }^{1}$ Department of Biochemistry, College of Medicine and Health Science, United Arab Emirates University, Al Ain, UAE

2 Department of Molecular Biology and Genetics, Democritus University of Thrace, Alexandroupolis, Greece

${ }^{3}$ Department of Biochemistry, Weill Cornell Medical College, Cornell University, New York, NY, USA

${ }^{4}$ Department of Anatomy, College of Medicine and Health Science, United Arab Emirates University, Al Ain, UAE

${ }^{5}$ College of Pharmacy and Sharjah Institute for Medical Research, University of Sharjah, Sharjah, UAE

${ }^{6}$ Department of Anatomy, Faculty of Medicine, King Abdulaziz University, Jeddah, Saudi Arabia

7 Faculty of Medicine, King Abdel Aziz University, Jeddah, Saudi Arabia

\section{Edited by:}

Edward James Calabrese, University

of Massachusetts/Amherst, USA

\section{Reviewed by:}

Kenjiro Ono, Kanazawa University

Graduate School of Medical

Science, Japan

Gjumrakch Aliev, GALLY

International Biomedical Research, USA

\section{*Correspondence:}

Omar M. A. El-Agnaf, Department of Biochemistry, College of Medicine and Health Science, United Arab Emirates University, Al Ain, UAE e-mail: o.elagnaf@uaeu.ac.ae
The aggregation of $\alpha$-synuclein ( $\alpha$-syn) is considered the key pathogenic event in many neurological disorders such as Parkinson's disease (PD), dementia with Lewy bodies and multiple system atrophy, giving rise to a whole category of neurodegenerative diseases known as synucleinopathies. Although the molecular basis of $\alpha$-syn toxicity has not been precisely elucidated, a great deal of effort has been put into identifying compounds that could inhibit or even reverse the aggregation process. Previous reports indicated that many phenolic compounds are potent inhibitors of $\alpha$-syn aggregation. The aim of the present study was to assess the anti-aggregating effect of gallic acid (GA) (3,4,5-trihydroxybenzoic acid), a benzoic acid derivative that belongs to a group of phenolic compounds known as phenolic acids. By employing an array of biophysical and biochemical techniques and a cell-viability assay, GA was shown not only to inhibit $\alpha$-syn fibrillation and toxicity but also to disaggregate preformed $\alpha$-syn amyloid fibrils. Interestingly, GA was found to bind to soluble, non-toxic oligomers with no $\beta$-sheet content, and to stabilize their structure. The binding of GA to the oligomers may represent a potential mechanism of action. Additionally, by using structure activity relationship data obtained from fourteen structurally similar benzoic acid derivatives, it was determined that the inhibition of $\alpha$-syn fibrillation by GA is related to the number of hydroxyl moieties and their position on the phenyl ring. GA may represent the starting point for designing new molecules that could be used for the treatment of PD and related disorders.

Keywords: $\alpha$-synuclein, Parkinson's disease, gallic acid, aggregation, amyloid fibrils, drug discovery

\section{INTRODUCTION}

Parkinson's disease (PD) is a neurodegenerative disorder, the incidence of which rises sharply after the fifth decade of life, affecting 1.4 and $3.4 \%$ of the population at 55 and 75 years of age, respectively (Wood, 1997). PD affects a region of the brain known as the substantia nigra, resulting in a dramatic loss of dopaminergic neurons and a concomitant plummeting in dopamine levels in striatum. As a consequence, PD is manifested by a plethora of clinical symptoms, with the most prominent being impaired motor activity, muscle rigidity, and resting tremor. Neuropathologically, PD is characterized by the presence of intracellular inclusions known as Lewy bodies (LBs), the main constituent of which are $\alpha$-synuclein ( $\alpha$-syn) fibrils (Spillantini et al., 1997; El-Agnaf et al., 1998). Moreover, accumulated results over the last few decades show that mitochondrial dysfunction followed by oxidative stress are both associated with neurodegenerative diseases (Leuner et al., 2007; Schmitt et al., 2012).
Although $\alpha$-syn belongs to the family of natively unfolded proteins, which demonstrate little or no ordered structure, the protein has the intrinsic propensity to fibrillate, giving rise to insoluble fibrils similar to the ones detected in LBs (Hashimoto et al., 1998; Giasson et al., 1999; Conway et al., 2000; Serpell et al., 2000; Greenbaum et al., 2005). Additionally, five missense mutations in the $\alpha$-syn gene (SNCA), namely A53T (Polymeropoulos et al., 1997), A30P (Krüger et al., 1998), E46K (Zarranz et al., 2004), H50Q (Appel-Cresswell et al., 2013) and G51D (Kiely et al., 2013), have been linked to severe inherited forms of PD, while duplications and triplications of SNCA lead to autosomal dominant PD in a gene dosage-dependent fashion (Singleton et al., 2003; Ibáñez et al., 2004). Moreover, transgenic animal and Drosophila models expressing either wild-type or mutant human $\alpha$-syn also develop fibrillar inclusions and a Parkinsonian phenotype (Feany and Bender, 2000; Masliah et al., 2000; Kahle et al., 2001; Imai et al., 2011; Cannon et al., 2013). Overexpression of $\alpha$-syn, especially its mutant forms, is thought to enhance the 
vulnerability of neurons to DA-induced cell death through an excessive generation of intracellular ROS (Junn and Mouradian, 2002). Many evidences point to the key role of $\alpha$-syn in the regulation and biosynthesis of DA, the loss of $\alpha$-syn function as a consequence to its aggregation lead to selective disrupt DA homeostasis and negatively affect dopaminergic neuron survival (Perez et al., 2002). It is noteworthy that the fibrillation of $\alpha$-syn is implicated in the development of a series of neurodegenerative diseases, including multiple system atrophy (MSA) and dementia with Lewy bodies (DLB), which are collectively referred to as synucleinopathies (Spillantini and Goedert, 2000; Trojanowski and Lee, 2003). Mitochondrial pathophysiology aggressively promotes neuronal dysfunction and loss of synaptic viability, leading ultimately to neurodegeneration (Perier and Vila, 2012), and accumulated evidence from both in vitro and in vivo studies, postulates a major pathogenic role for a $\alpha$-syn in mitochondrial dysfunction, thereby providing a link between protein aggregation, mitochondrial damage, and neurodegeneration (reviewed in Camilleri and Vassallo, 2014). Taken together, these findings indicate a central role for $\alpha$-syn aggregation in PD pathogenesis. $\alpha$-Syn aggregation proceeds through several key intermediate stages, with monomeric $\alpha$-syn first assembling into oligomeric forms that gradually generate insoluble amyloid fibrils. Because $\alpha$-syn aggregation plays a crucial role in PD pathogenesis and related synucleinopathies, intensive effort has been put into identifying compounds that could block or even reverse the aggregation process. Over the years, polyphenols, a set of more than 8000 compounds that contain one or more phenolic rings, have emerged as potent amyloid inhibitors, interfering with the in vitro fibril assembly of many amyloidogenic proteins including $\alpha$-syn, $\beta$-amyloid $(\mathrm{A} \beta)$, tau-protein and prions (reviewed in Porat et al., 2006).

Gallic acid (GA) is a phenolic acid. Phenolic acids constitute a group of compounds, which are derived from benzoic acid and cinnamic acid, giving rise to hydroxybenzoic acids and hydrocinnamicacids, respectively. GA (3,4,5-trihydroxybenzoic acid) is a benzoic acid derivative that can be found in almost all plants, with the highest GA contents detected in gallnuts, witch hazel, pomegranate, berries such as blackberry and raspberry, sumac, tea leaves and oak bark. GA can also be isolated from the roots of Radix Paeoniae (white-flowered peony), which is commonly used to treat vascular and liver diseases in traditional Chinese medicine (Ho and Hong, 2011). It has been reported that GA possesses antioxidant (Kim, 2007), anti-inflammatory (Kroes et al., 1992) and anti-viral (Kreis et al., 1990) properties, and a well-documented anti-cancer activity (Yang et al., 2000; Liu et al., 2011; Ho et al., 2013). Recently, GA has been reported to act as a potent antioxidant and free radical scavenger in a rat PD model (Sameri et al., 2011). Additionally, GA was shown to efficiently inhibit $\alpha$-syn and $\mathrm{A} \beta$ aggregation and toxicity in vitro (Bastianetto et al., 2006; Di Giovanni et al., 2010).

The aim of the present study was to systematically assess the ability of GA to (a) inhibit $\alpha$-syn oligomerization and fibrillation, (b) block $\alpha$-syn-induced toxicity and (c) disaggregate preformed $\alpha$-syn fibrils. To gain insight of the mechanism of action of GA against $\alpha$-syn aggregation and toxicity and to establish a structure-activity relationship, we assessed the anti-fibrillogenic effect of eleven different hydroxybenzoic acid derivatives with chemical structures similar to GA. The selection of the phenolic acids was based on the number of the hydroxyl moieties attached to the phenyl ring. To further investigate the role of hydroxyl groups in the inhibitory activity of phenolic acids, we also included and assessed the effect of three different benzoic acid derivatives that have fluorides and methoxy groups instead of hydroxyl moieties.

\section{MATERIALS AND METHODS EXPRESSION AND PURIFICATION OF RECOMBINANT HUMAN $\alpha$-SYN}

The GST- $\alpha$-syn fusion construct in pGEX-4T1 vector (kindly provided by Dr. Hyangshuk Rhim, The Catholic University College of Medicine, Seoul, Korea) was inserted into E.coli BL21bacteria for protein expression by heat shock transformation. The transformed bacteria were grown in LB medium supplemented with $0.1 \mathrm{mg} / \mathrm{ml}$ ampicillin at $37^{\circ} \mathrm{C}$ in an orbital shaker until the OD600 was 0.5 . GST- $\alpha$-syn expression was then induced by adding $0.5 \mathrm{mM}$ IPTG (Sigma-Aldrich Chemie GmbH, Germany), and the culture was incubated for $2 \mathrm{~h}$ at $37^{\circ} \mathrm{C}$. The cells were harvested by 15 min centrifugation at $9000 \times \mathrm{g}$ (Beckman Coulter, Avanti J-26 $\mathrm{XP})$. The pellets were resuspended in lysis buffer $(50 \mathrm{mM}$ Tris$\mathrm{HCl}, \mathrm{pH}$ 7.4, $150 \mathrm{mM} \mathrm{NaCl}, 2$ mM EDTA, 1\% NP-40, 0.1\% DTT), and the lysed pellets were shaken for $10 \mathrm{~min}$ at room temperature. The lysed pellets were then subjected to 6 freeze-thaw cycles in liquid nitrogen and a $37^{\circ} \mathrm{C}$ water bath. The lysates were centrifuged further at $27,000 \times \mathrm{g}$ for $15 \mathrm{~min}$. The supernatant was kept for purification with affinity chromatography. Glutathione sepharose $4 \mathrm{~B}$ beads (Amersham, Sweden) were centrifuged at $500 \times \mathrm{g}$ at $4^{\circ} \mathrm{C}$ for $8 \mathrm{~min}$ to remove the storage buffer. The beads were then washed with $20 \mathrm{ml}$ cold PBS followed by spinning at $500 \times \mathrm{g}$ at $4^{\circ} \mathrm{C}$ for $10 \mathrm{~min}$. The cell lysate was mixed with the washed beads and incubated for $1 \mathrm{~h}$ at room temperature, followed by centrifugation at $500 \times \mathrm{g}$ at $4^{\circ} \mathrm{C}$ for $8 \mathrm{~min}$. The beads were then washed twice with column washing buffer $(50 \mathrm{mM}$ Tris- $\mathrm{HCl}, 150 \mathrm{mM}$ $\mathrm{NaCl}, 10 \mathrm{mM}$ EDTA, 1\% Triton X-100, pH 8.0), twice with $50 \mathrm{mM}$ Tris- $\mathrm{HCl}, \mathrm{pH} 8.0$ and once with $1 \mathrm{X}$ PBS. The washed beads were resuspended in $5 \mathrm{ml}$ of $1 \mathrm{X}$ PBS. The GST tag, which interacts with glutathione beads, was cleaved by thrombin ( 1 unit/ $\mu$ l human plasma thrombin, Sigma). The thrombin-catalyzed reaction was incubated overnight at room temperature with continuous mixing. The mixture was then incubated for $5 \mathrm{~min}$ at $37^{\circ} \mathrm{C}$ and centrifuged for $8 \mathrm{~min}$ at $500 \times \mathrm{g}$ at $4^{\circ} \mathrm{C}$. Benzamidinesepharose beads (Amersham, Sweden) were used to remove the thrombin from the solution. Pure $\alpha$-syn was collected by centrifugation at $500 \times \mathrm{g}$ for $8 \mathrm{~min}$ at $4^{\circ} \mathrm{C}$. The $\alpha$-syn concentration was estimated using the BCA assay (Pierce Biotechnology, Rockford, IL).

\section{AGGREGATION OF $\alpha$-SYN IN VITRO}

Stock solutions of the tested compounds $(10 \mathrm{mM})$ were prepared in DMSO. Solutions of lower concentrations were prepared by diluting the stock solutions to final concentrations of $25-100 \mu \mathrm{M}$. The amount of DMSO in the final samples was $1 \%$. Samples of $25 \mu \mathrm{M}$ (unless otherwise stated) $\alpha$-syn in PBS were aged either alone or with phenolic acids at various molar ratios (phenolic acids to protein molar ratios of $4: 1,2: 1$, and $1: 1$ ). The samples were placed in $1.5 \mathrm{ml}$ sterile polypropylene tubes, sealed with 
parafilm and incubated at $37^{\circ} \mathrm{C}$ for 5 days with continuous shaking at $800 \mathrm{rpm}$ in a Thermomixer (Eppendorf). Samples were collected at regular time points. Thioflavin-S (Th-S) fluorescence was measured immediately, while the rest of the samples were stored at $-80^{\circ} \mathrm{C}$ until needed for further analysis.

\section{THIOFLAVIN-S (TH-S) FLUORESCENCE ASSAY}

$\alpha$-Syn fibril formation was monitored by Th-S binding. Th-S is a fluorescent dye that interacts with fibrils in a $\beta$-sheet structure. Each sample $(10 \mu \mathrm{l})$ was mixed with $40 \mu \mathrm{l}$ of Th-S $(25 \mu \mathrm{M})$ in PBS. Fluorescence was measured in a 384-well, non-treated, black micro-well plate (Nunc, Denmark) using a Victor X3 2030 (Perkin Elmer) microplate reader with excitation and emission wavelengths of 450 and $486 \mathrm{~nm}$, respectively. To allow for background fluorescence, the fluorescence intensity of a blank well containing only PBS solution was subtracted from all readings.

\section{TRANSMISSION ELECTRON MICROSCOPY (TEM)}

Electron microscopy images were produced from $\alpha$-syn aged in the presence or absence of GA. The samples $(5 \mu \mathrm{L})$ were deposited on Formvar-coated 400 mesh copper grids, fixed briefly with $0.5 \%$ glutaraldehyde $(5 \mu \mathrm{l})$, negatively stained with $2 \%$ uranyl acetate (Sigma-Aldrich) and examined in a Philips CM-10 TEM electron microscope.

\section{IMMUNOBLOTTING}

Samples (20 ng) of $\alpha$-syn incubated alone or with GA were mixed with loading sample buffer $(250 \mathrm{mM}$ Tris- $\mathrm{HCl}, \mathrm{pH} 6.8,30 \%$ glycerol, $0.02 \%$ bromophenol blue) without SDS or boiling and then separated on $1 \mathrm{~mm} 15 \%$ SDS-PAGE gels. The separated proteins were transferred to nitrocellulose membranes $(0.45 \mu \mathrm{m}$, WhatmanGmbh-Germany) at $90 \mathrm{~V}$ for $80 \mathrm{~min}$. The membranes were boiled for $5 \mathrm{~min}$ in PBS and then blocked for $1 \mathrm{~h}$ with $5 \%$ non-fat milk prepared in PBS-Tween-20 (0.05\% PBST). The membranes were incubated overnight at $4^{\circ} \mathrm{C}$ with the primary antibody, namely the mouse monoclonal anti- $\alpha$-syn (211) that recognizes human $\alpha$-syn (121-125) (Santa Cruz Biotechnology, USA), at a dilution of 1:1000. The membranes were then washed several times with PBST, followed by incubation with HRPconjugated goat anti-mouse antibody (Dako Ltd., Ely, UK) at a dilution of 1:70,000 for $60 \mathrm{~min}$ at room temperature and gentle agitation. The membranes were then extensively washed for $25 \mathrm{~min}$. The immunoreactive bands were visualized with a SuperSignal West FemtoChemiluminescent Substrate Kit (Pierce, Rockford, USA) according to the manufacturer's instructions.

\section{IMMUNOASSAY FOR MEASURING OLIGOMERIC $\alpha$-SYN}

A384-well ELISA plate (NuncMaxisorb,Nunc, Denmark) was coated with $1 \mu \mathrm{g} / \mathrm{ml}$ of non-biotinylated mouse monoclonal anti- $\alpha$-syn antibody [mAb, 211 -recognizes amino acid residues 121-125 of human $\alpha$-syn (Santa Cruz Biotechnology, California, USA)] diluted in $200 \mathrm{mM} \mathrm{NaHCO}_{3}$, pH 9.6 (50 $\mu \mathrm{l} /$ well) and incubated overnight at $4^{\circ} \mathrm{C}$. The plate was then washed 4 times with PBST and blocked with $100 \mu \mathrm{l} /$ well of blocking buffer (5\% gelatin from cold water fish skin, $0.05 \%$ Tween 20 in $1 \mathrm{X} \mathrm{PBS} \mathrm{pH} 7.4$ ) for $2 \mathrm{~h}$ at $37^{\circ} \mathrm{C}$. After washing 4 times with PBST, $50 \mu \mathrm{l}$ of the samples were dispensed in each well, and each sample was tested in duplicate. The plates were then incubated at $37^{\circ} \mathrm{C}$ for another $3 \mathrm{~h}$.
After washing 4 times with PBST, $50 \mu \mathrm{l}$ of biotinylated 211 antibody diluted in blocking buffer to a concentration of $0.4 \mu \mathrm{g} / \mathrm{ml}$ was added and incubated at $37^{\circ} \mathrm{C}$ for $2 \mathrm{~h}$. The wells were washed 4 times with PBST and incubated with $50 \mu \mathrm{l} /$ well of ExtravidinPeroxidase (Sigma-Aldrich, GmbH- Germany) diluted 1:7500 in blocking buffer and incubated for $1 \mathrm{~h}$ at $37^{\circ} \mathrm{C}$. The wells were then washed 4 times with PBST before adding SuperSignal ELISA Femto Maximum Sensitivity Substrate (Pierce Biotechnology, Rockford, USA) (50 $\mu \mathrm{L} /$ well). The chemiluminescence in relative light units was immediately measured using amicroplate reader (Perkin Elmer).

\section{CULTURE OF BE (2)-M17 HUMAN NEUROBLASTOMA CELLS}

BE (2)-M17 human neuroblastoma cells were routinely cultured in Dulbecco's MEM/Nutrient Mix F-12 (1:1) (Gibco BRL, Rockville, MD) supplemented with $15 \%$ fetal bovine serum and $1 \%$ penicillin-streptomycin $(100 \mathrm{U} / \mathrm{ml}$ penicillin, $100 \mathrm{mg} / \mathrm{ml}$ streptomycin). The cells were maintained at $37^{\circ} \mathrm{C}$ in a humidified incubator with 5\% CO2/95\% air.

\section{CELL VIABILITY ASSAY}

To evaluate the cell viability of BE (2)-M17 human neuroblastoma cells treated with $\alpha$-syn aggregates, the 3-(4,5-dimethylthiazol2-yl)-2,5-diphenyltetrazolium bromide (MTT) reduction assay was performed as described previously (Mosmann, 1983). Briefly, BE (2)-M17 cells in DMEM medium were plated at a density of 15,000 cells $/ 100 \mu \mathrm{l} /$ well in a 96 -well plate. After $24 \mathrm{~h}$, the medium was replaced with $200 \mu \mathrm{l}$ of OPTI-MEM (Gibco BRL) serum-free medium containing either $\alpha$-syn aged in the presence or absence of GA or pre-aged $\alpha$-syn incubated with different concentrations of GA. Samples were diluted in OPTI-MEM to obtain the desired concentration. Cells were then returned into the incubator and incubated for $48 \mathrm{~h}$. A total of $20 \mu \mathrm{l}$ of MTT $(6 \mathrm{mg} / \mathrm{ml})$ in PBS was added to each well, and the plate was incubated at $37^{\circ} \mathrm{C}$ for $4.5 \mathrm{~h}$. The medium-MTT solution was removed, cell lysis buffer (100 $\mu \mathrm{l} /$ well; 15\% (w/v) SDS/50\% (v/v) $\mathrm{N}, \mathrm{N}$-dimethylformamide, $\mathrm{pH}$ 4.7) was added, and the plate was incubated overnight at $37^{\circ} \mathrm{C}$. Absorbance values at $590 \mathrm{~nm}$ were determined using a plate reader (Perkin Elmer).

\section{CONGO RED BINDING ASSAY}

Congo red $(20 \mu \mathrm{M})$ was dissolvedin PBS ( $\mathrm{pH} 7.4)$ and filtered through a $0.45 \mu \mathrm{m}$ filter. Samples of $\alpha$-syn $(5 \mu \mathrm{M})$, aged alone or with GA at different molar ratios, were mixed with Congo red (final concentration $5 \mu \mathrm{M}$ ), and the reaction samples were thoroughly mixed. The UV absorbance spectrum was recorded from 400 to $600 \mathrm{~nm}$ in a spectrophotometer (DU800 , Beckman-Coulter) using $10 \mathrm{~mm}$ quartz cuvettes (Hellma Analytics-Germany). Monomeric $\alpha$-syn and Congo red alone were used as negative controls.

\section{$\alpha$-SYN DISAGGREGATION ASSAY}

Recombinant $\alpha$-syn dissolved in sterilized PBS ( $\mathrm{pH}$ 7.4) was aggregated at a concentration of $25 \mu \mathrm{M}$ as indicated above. Briefly, the $\alpha$-syn samples were placed in $1.5 \mathrm{ml}$ sterile polypropylene tubes sealed with parafilm and incubated at $37^{\circ} \mathrm{C}$ for 5 days, with continuous shaking at $800 \mathrm{rpm}$ in a Thermomixer (Eppendorf). The resulting aggregated $\alpha$-syn was incubated either 
alone or with GA at various molar ratios (GA: $\alpha$-syn molar ratios $6: 1,4: 1$, and 2:1). It should be noted that for the purpose of the experiment, the concentration of $\alpha$-syn was assumed to be the same as that of the fresh $\alpha$-syn. The samples were incubated at $37^{\circ} \mathrm{C}$ for 3 days on a thermomixer with continuous shaking at $800 \mathrm{rpm}$. Samples were collected at regular time points, and Th-S fluorescence was measured immediately.

\section{SEEDING POLYMERIZATION ASSAY}

The aggregation of monomeric $\alpha$-syn with or without seeding was carried out as described elsewhere (Di Giovanni et al., 2010). Mature $\alpha$-syn fibrils were fragmented by sonication to obtain short fibrils, which were employed as 'seeds'. Briefly, $100 \mu \mathrm{M}$ monomeric $\alpha$-syn was seeded with $2 \mu$ Mseeds and incubated in the presence or absence of GA $(10 \mu \mathrm{M}$ and $50 \mu \mathrm{M})$ at $37^{\circ} \mathrm{C}$ for $6 \mathrm{~h}$ with continuous shaking. Fibrillation was monitored by Th-S binding as described above.

\section{PREPARING WT- $\alpha$-SYN OLIGOMERS}

Recombinant $\alpha$-syn dissolved in sterilized PBS ( $\mathrm{pH} 7.4$ ) was aggregated at a concentration of $100 \mu \mathrm{M}$. Sample was placed in $1.5 \mathrm{ml}$ sterile polypropylene tubes, sealed with parafilm and incubated at $37^{\circ} \mathrm{C}$ for 2 days, with continuous shaking at $800 \mathrm{rpm}$ in a Thermomixer (Eppendorf).

\section{NATIVE PAGE}

Samples of $\alpha$-syn oligomers, $\alpha$-syn: GA oligomers and $\alpha$-syn monomers (15 ng), were separated in 3-1\% Bis-Tris Native PAGE gel (Novox, Life technologies) according to the manufacturer's instructions. The separated proteins were transferred to PVDF membrane $(0.45 \mu \mathrm{m}$, Thermo Scientific) at $100 \mathrm{~V}$ for $120 \mathrm{~min}$, after soaking the methanol for $30 \mathrm{~s}$, and followed by soaking in $1 \mathrm{x}$ methanol free transfer buffer for $10 \mathrm{~min}$. The membrane then blocked for $1 \mathrm{~h}$ with $5 \%$ non-fat milk prepared in PBS-Tween-20 $(0.05 \%$ PBST $)$. The membrane were incubated overnight at $4{ }^{\circ} \mathrm{C}$ with the primary antibody, namely the mouse monoclonal anti$\alpha$-syn (211) that recognizes human $\alpha$-syn (121-125) (Santa Cruz Biotechnology, USA), at a dilution of 1:1000. The membranes were then washed several times with PBST, followed by incubation with HRP-conjugated goat anti-mouse antibody (Dako Ltd., Ely, UK) at a dilution of 1:120,000 for $60 \mathrm{~min}$ at room temperature and gentle agitation. The membranes were then extensively washed for $25 \mathrm{~min}$. The immunoreactive bands were visualized with a SuperSignal West FemtoChemiluminescent Substrate Kit (Pierce, Rockford, USA) according to the manufacturer's instructions.

\section{SIZE EXCLUSION CHROMATOGRAPHY (SEC) FOR SEPARATING $\alpha$-SYN OLIGOMERS AND MONOMERS}

SEC was carried out using an AKTA FPLC system (GE HealthcareSweden) and a superdex 200 column at $4^{\circ} \mathrm{C}$, in order to separate the oligomers generated from the aggregation of $\alpha$-syn with GA (GA: $\alpha$-syn molar ratio of 4:1). Monomeric $\alpha$-syn at a concentration of $100 \mu \mathrm{M}$ was aggregated in the presence of GA for 5 days as described above. At the end of the aggregation process, the sample was centrifuged for $45 \mathrm{~min}$ at $14,000 \times \mathrm{g}$ at $4^{\circ} \mathrm{C}$ generating a supernatant free from insoluble material. Prior to injecting $80 \%$ of the generated supernatant, the column was thoroughly equilibrated with SEC running buffer $(1 \times \mathrm{PBS}, \mathrm{pH} 7.4)$ and the flow rate was set to $0.1 \mathrm{ml} / \mathrm{min}(0.5 \mathrm{ml} /$ fraction $)$. $\alpha$-Syn elution was monitored at absorbance wavelengths of 215, 254, and $280 \mathrm{~nm}$. To determine the elution time of monomeric $\alpha$-syn, molecular weight standards (ferritin $440 \mathrm{kDa}$, aldolase $171 \mathrm{kDa}$, abmumin $68 \mathrm{kDa}$ and chymotrypsinogen $\mathrm{A} 25 \mathrm{kDa}$ ) and monomeric $\alpha$-syn were co-injected into the column and eluted at the same conditions mentioned above. The fractions eluting between 7-9 $\mathrm{ml} \mathrm{CV}$ were combined and labeled as oligomers (sample P1), whereas the fractions eluting in the $13-15 \mathrm{ml} \mathrm{CV}$ were combined and labeled as monomers (sample P2). The P1 and P2 fractions were further characterized by western blotting and TEM.

\section{UV SCANNING}

The P1 and P2 samples, representing the oligomeric and monomeric fractions of SEC, respectively, were concentrated using a speed vac (CentriVap, Labconco). Their protein content concentration was estimated by the BCA assay. The UV absorbance spectrum was recorded from 200 to $600 \mathrm{~nm}$ in a spectrophotometer (DU-800, Beckman-Coulter) using $10 \mathrm{~mm}$ quartz cuvettes (Hellma Analytics-Germany) and employing equal concentrations of both P1 and P2. Fresh monomeric $\alpha$-syn was used as negative control.

\section{NMR}

For NMR studies, recombinant $15 \mathrm{~N}$-labeled $\alpha$-syn was expressed and purified as previously described (Eliezer et al., 2001; Bussell and Eliezer, 2003), resuspended in PBS at pH 6.5. Twodimensional proton-nitrogen correlation spectra were acquired for $\alpha$-syn at $200 \mu \mathrm{M}$ concentration in the absence of GA and in the presence of increasing GA: $\alpha$-syn stoichiometries of $0.5: 1,1: 1$, $2: 1,5: 1$, and 10:1. Data were collected on a Varian $600 \mathrm{MHz}$ Unity Inova spectrometer equipped with a cold probe.

\section{RESULTS}

\section{THE EFFECT OF GA ON $\alpha$-SYN FIBRIL FORMATION}

To investigate the effect of GA on $\alpha$-syn fibrillation, $25 \mu \mathrm{M}$ of $\alpha$ syn was incubated with GA at varying molar ratios of GA: $\alpha$-syn of $4: 1,2: 1$, and $1: 1$ for a period of 6 days. GA inhibited the formation of $\alpha$-syn fibrils in a concentration-dependent manner as indicated by the Th-S assay (Figure 1A). Taking into account the lack of Th$S$ signal at the 4:1 ratio, GA exhibited an excellent inhibitory effect on $\alpha$-syn fibrillation, inhibiting it completely during the 6-day incubation (Figure 1A). At a 2:1 ratio, GA also suppressed the formation of fibrils to a great extent, and the Th-S signal was detected only after 4 days of incubation, while after 6 days, GA reduced the Th-S counts to approximately one fifth of the control counts (Figure 1A). Even at a 1:1 ratio, GA hindered the fibrillation of $\alpha$-syn but to a smaller extent than the other ratios (Figure 1A). The anti-fibrillogenic activity of GA was further assessed by the Congo red binding assay. Congo red (CR) is a dye with high affinity for amyloid fibrils. The absorption maximum of $\mathrm{CR}$ alone $(5 \mu \mathrm{M})$ was $490 \mathrm{~nm}$, while when incubated with $\alpha$-syn amyloid fibrils, the absorption maximum shifted to $508 \mathrm{~nm}$ (Figure 1B). This shift in the peak absorption wavelength represents the binding of CR to the $\beta$-sheet-rich fibrils. However, this prominent shift 

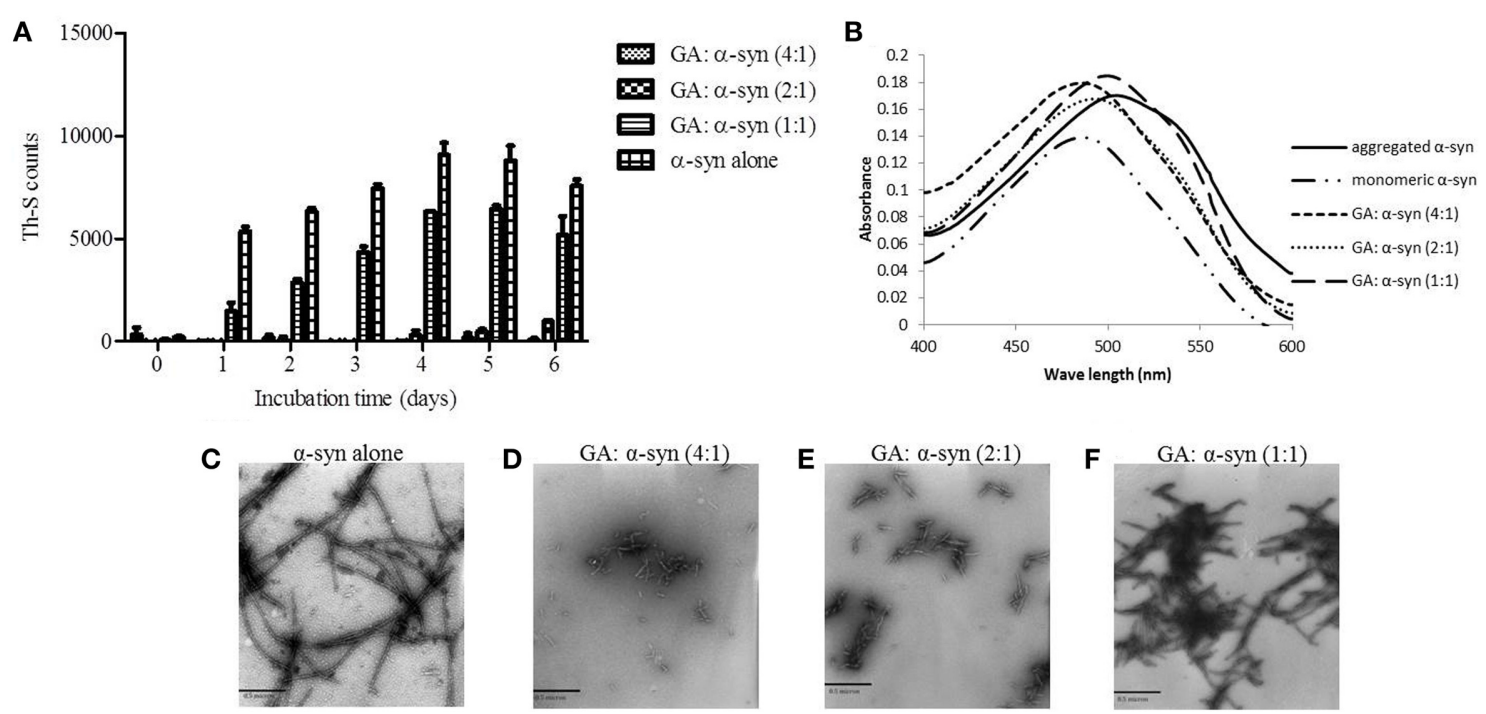

FIGURE 1 | GA inhibits $\alpha$-syn fibrillation in a concentration-dependent manner. (A) Samples of $\alpha$-syn $(25 \mu \mathrm{M})$ were incubated alone or in the presence of GA (molar ration of GA: $\alpha$-syn 4:1, 2:1, 1:1) for 6 days with continuous shaking at $37^{\circ} \mathrm{C}$. Fibril formation was estimated by Th-S fluorescence. The assay was performed in triplicate (average of triplicate measurement \pm standard deviation). (B) Congo red binding for samples of $\alpha$-syn $(25 \mu \mathrm{M})$ incubated alone or in the presence of GA (molar ratio of GA: $\alpha$-syn $4: 1,2: 1,1: 1)$ for 6 days with continuous shaking at $37^{\circ} \mathrm{C}$. Samples of $\alpha$-syn $(5 \mu \mathrm{M})$ aged alone or with GA were mixed with Congo red at a final concentration of $5 \mu \mathrm{M}$. The reaction samples were thoroughly mixed and placed in a $10 \mathrm{~mm}$ quartz cuvette. The UV absorbance spectra were recorded from 400 to $600 \mathrm{~nm}$. C-F. Electron microscopy images of negatively stained samples of $\alpha$-syn $(25 \mu \mathrm{M})$ aged alone or in the presence of GA (molar ratio of GA: $\alpha$-syn $4: 1,2: 1,1: 1)$ for 6 days with continuous shaking at $37^{\circ} \mathrm{C}$. (C) Aged $\alpha$-syn alone. (D) $\alpha$-Syn aged in the presence of GA at a molar ratio of GA: $\alpha$-syn 4:1. (E) $\alpha$-Syn aged in the presence of GA at a molar ratio of GA: $\alpha$-syn $2: 1$. (F) $\alpha$-Syn aged in the presence of GA at a molar ratio of GA: $\alpha$-syn $1: 1$. Scale bar, $500 \mathrm{~nm}$. was not observed for the fresh $\alpha$-syn sample or when $\alpha$-syn was aged in the presence of GA (Figure 1B), indicating that GA inhibited the formation of structures with a $\beta$-sheet conformation. In fact, GA blocked the formation of $\beta$-sheets in a dose-dependent manner, similar to the Th-S results. It was observed that the lower the GA concentration, the more prominent the peak absorbance wavelengths shift was $(485,493$, and $500 \mathrm{~nm}$ for 100,50 , and $25 \mu \mathrm{M}$ GA, respectively). Electron microscopy images also confirmed that GA not only affected the extent of fibrillation in a dose-dependent fashion but also the morphology of $\alpha$-syn fibrils. Indeed, instead of forming dense meshes of long fibrils (longer than $1 \mu \mathrm{m}$ ) as observed in aged $\alpha$-syn alone (Figure 1C), $\alpha$-syn aged in the presence of $100 \mu \mathrm{M}$ (Figure 1D) and $50 \mu \mathrm{M}$ of GA (Figure 1E) generated thin, sheared fibrils that were approximately $0.1-0.2 \mu \mathrm{m}$ in length. $\alpha$-Syn aged in the presence of $25 \mu \mathrm{M}$ GA (Figure 1F) formed longer fibrils (approximately $0.5 \mu \mathrm{m}$ long), which were arranged in dense networks.

\section{THE EFFECT OF GA ON $\alpha$-SYN OLIGOMERIZATION (EARLY AGGREGATES)}

To evaluate the effect of GA on $\alpha$-syn oligomer formation, samples of $\alpha$-syn aged alone or in the presence of GA at different molar ratios were assessed for their oligomeric content by oligomer-specific ELISA and immunoblotting. For the detection of $\alpha$-syn oligomeric species in the samples, we employed a novel ELISA developed in our laboratory (El-Agnaf et al., 2006), which specifically recognizes the oligomeric species present in the samples. The ELISA results (Figure 2A) indicated that GA could only inhibit the formation of $\alpha$-syn oligomers when employed at the highest concentration, namely $100 \mu \mathrm{M}$. Interestingly, at lower concentrations, GA appeared to enhance $\alpha$-syn oligomerization compared to the control (Figure 2A). The samples of $\alpha$-syn aged in the presence of 25 and $50 \mu \mathrm{M}$ GA exhibited gradually increasing oligomeric content, which was much higher than the control (Figure 2A).

Similarly, immunoblotting of the same samples (Figure 2B) indicated a decrease in the oligomeric species when $\alpha$-syn was aged with $100 \mu \mathrm{M}$ GA (Figure 2B), while there was a characteristic increase in the band corresponding to dimeric $\alpha$-syn (Figure 2B). The same sample was also characterized by the presence of strong monomeric and trimeric (approximately $50 \mathrm{kDa}$ ) bands and the absence of the bands corresponding to larger $\alpha$-syn aggregates with high molecular weight. However, in the presence of 50 and $25 \mu \mathrm{M}$ GA, the bands corresponding to oligomeric species were much stronger, with a concentration-dependent decrease in the bands corresponding to monomeric, dimeric and trimeric species. Additionally, the intensity of the band corresponding to larger $\alpha$-syn aggregates increased in a dose-dependent manner. These data indicate that GA at low concentrations may have the ability to stabilize $\alpha$-syn oligomers.

\section{THE EFFECT OF GA ON PREFORMED $\alpha$-SYN AMYLOID FIBRILS}

Given that GA was shown to be such an effective inhibitor of $\alpha$-syn fibrillation, we investigated whether it could also reverse fibrillation. Therefore, $25 \mu \mathrm{M}$ of preformed $\alpha$-syn fibrils were incubated at $37^{\circ} \mathrm{C}$ in the presence of GA at molar ratios of GA: $\alpha$ syn of $6: 1,4: 1$, and $2: 1$ for a period of $48 \mathrm{~h}$. By measuring the Th-S 


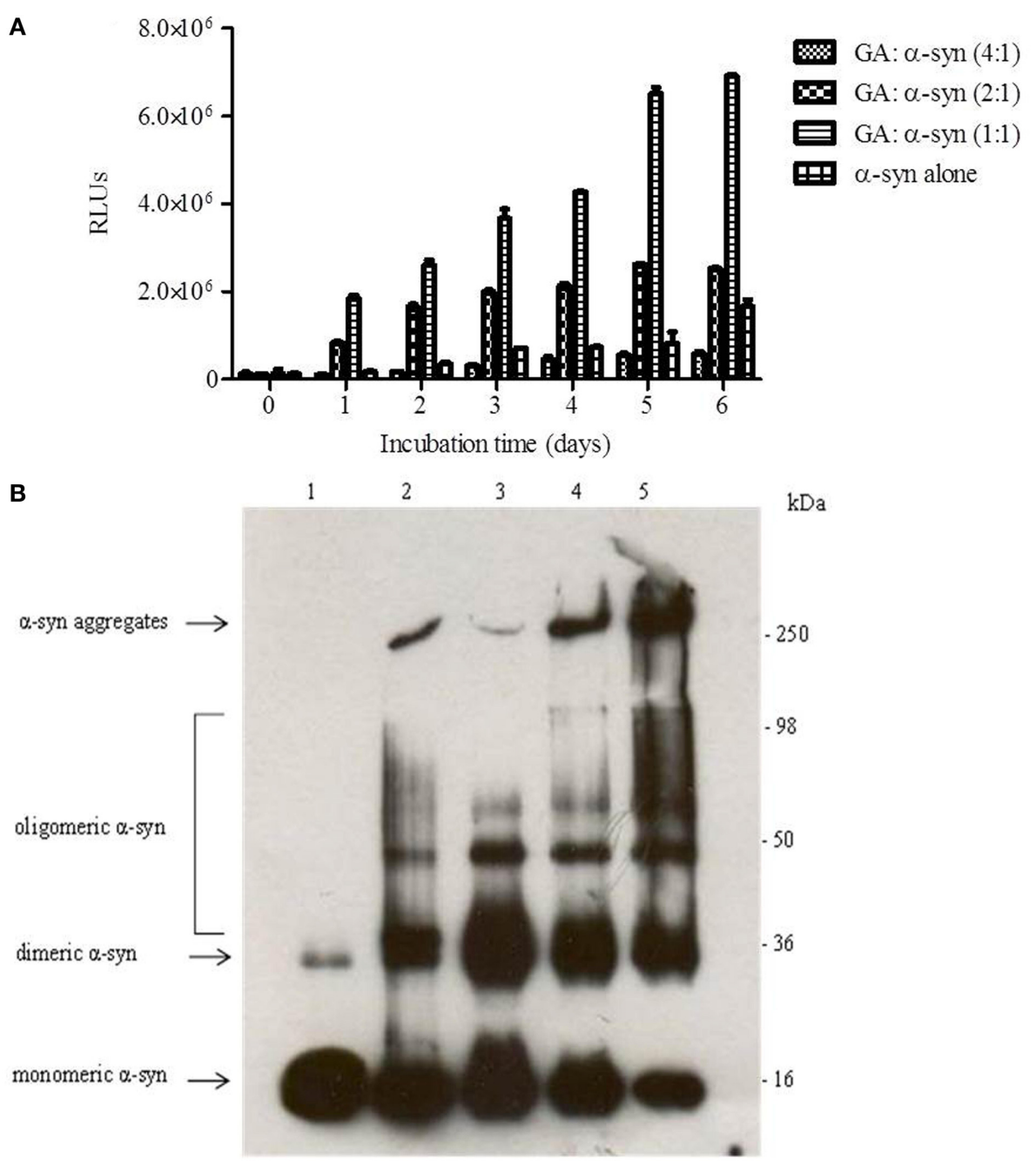

FIGURE 2 | GA inhibits $\alpha$-syn oligomerization at a high concentration, but at lower concentrations it promotes oligomerization. (A) Samples of $\alpha$-syn $(25 \mu \mathrm{M})$ aged alone or in the presence of GA at different molar ratios for 6 days with continuous shaking at $37^{\circ} \mathrm{C}$ were assessed for their ability to inhibit the formation of oligomers by the oligomeric ELISA assay. The assay was performed in duplicate (average of duplicate measurements \pm standard deviations). (B) Immunoblot analysis of the effect of GA on $\alpha$-syn oligomerization. Fresh or aged $\alpha$-syn samples alone or in the presence of $\mathrm{GA}$ at molar ratios of GA: $\alpha$-syn 1:1, 2:1 and $4: 1$ incubated for 6 days with continuous shaking at $37^{\circ} \mathrm{C}$ were separated by electrophoresis in a15\% SDS-PAGE gel. Lane 1: fresh $\alpha$-syn; lane 2: aged $\alpha$-syn; lane 3: GA: $\alpha$-syn molar ratio of $4: 1$, lane 4: GA: $\alpha$-syn molar ratio $2: 1$ and lane $5:$ GA: $\alpha$-syn molar ratio 1:1. fluorescence counts (Figure 3A), we estimated the fibril content at the indicated time points. At time 0 , the Th-S counts were approximately 8000 for all samples (Figure 3A). The $\alpha$-syn fibrils that were incubated alone continued to aggregate further, as indicated by the increase in Th-S counts (Figure 3A). However, the $\alpha$-syn fibrils that were incubated in the presence of GA disaggregated over time as shown by the decrease in the Th-S counts (Figure 3A). It is noteworthy that after $24 \mathrm{~h}$ of incubation, $\alpha$ syn fibrils incubated without GA gave approximately 18,000 Th-S counts, while the fibrils incubated with all tested concentrations of GA produced less than 2000 Th-S counts (Figure 3A). Thus, GA disaggregated preformed $\alpha$-syn fibrils in a dose-dependent fashion.

After $48 \mathrm{~h}$ of incubation, the samples were also tested for their CR binding (Figure 3B). As expected, $\alpha$-syn fibrils incubated in the presence of GA produced spectra with a peak absorbance wavelength that was slightly shifted compared to the controls, indicating minimal $\beta$-sheet content. In contrast, the fibrils of $\alpha$-syn that were incubated alone produced a peak absorbance at $550 \mathrm{~nm}$, indicating a high $\beta$ sheet content (Figure 3B). These findings were confirmed by EM (Figure 3C). All samples at time 0 were characterized by the presence of dense meshes of fibrils longer than $0.5 \mu \mathrm{m}$. However, the $\alpha$-syn fibrils incubated in the presence of GA gradually became thinner and shorter and appeared fragmented, isolated and scarce, unlike the control, which continued to demonstrate networks of long fibrils (Figure 3C). Furthermore, similar results were obtained when the experiment was performed using $\alpha$-syn fibrils after reaching plateau in Th-S assay (Figure S1). 

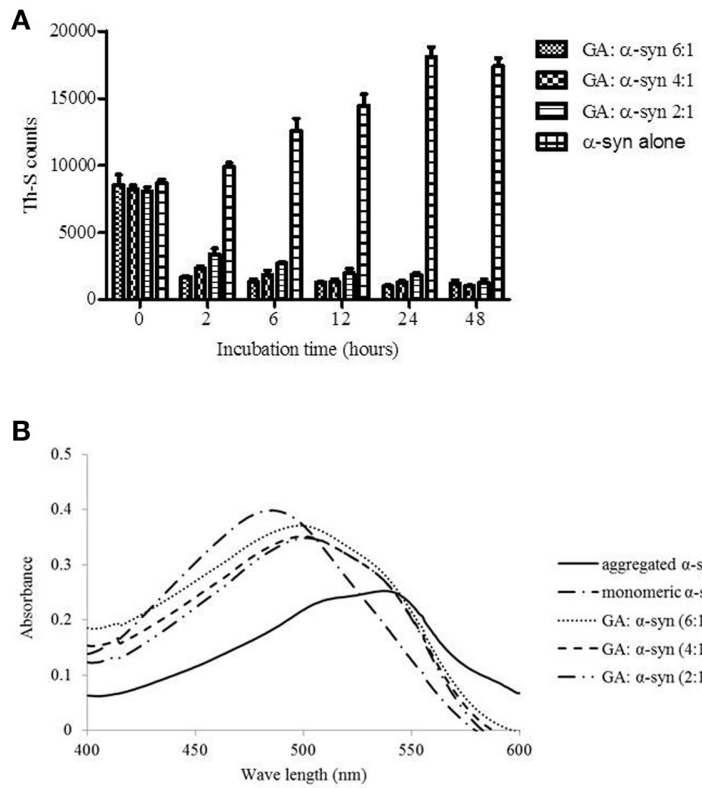

aggregated $\alpha$-syn $-\cdot-$ monomeric $\alpha$-syn ........ GA: $\alpha-\operatorname{syn}(6: 1)$ - - -GA: $\alpha$-syn $(4: 1)$ $-\cdots$ GA: $\alpha-\operatorname{syn}(2: 1)$

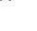

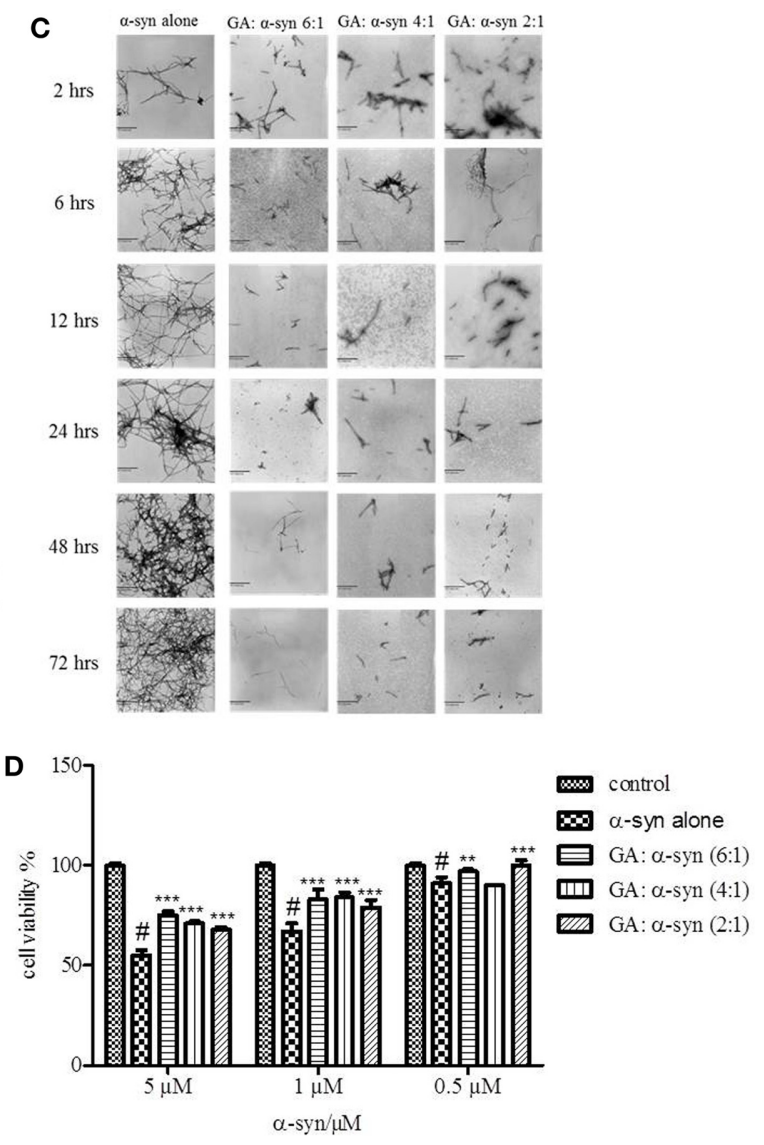

samples of pre-aged $\alpha$-syn $(25 \mu \mathrm{M})$ incubated alone or in the presence of GA for $72 \mathrm{~h}$ with continuous shaking at $37^{\circ} \mathrm{C}$. 1. $\alpha$-Syn aged alone. 2. $\alpha$-Syn aged in the presence of GA at a GA: $\alpha$-syn molar ratio of $6: 1$. 3. $\alpha$-Syn aged in the presence of GA at a GA: $\alpha$-syn molar ratio of 4:1. 4. $\alpha$-Syn aged in the presence of GA at a GA: $\alpha$-syn molar ratio of 2:1. Scale bar, $500 \mathrm{~nm}$. (D) The disaggregation of preformed $\alpha$-syn fibrils by GA generated species that were less toxic to the cells. The viability of BE (2)-M17 human neuroblastoma cells was assessed by the MTT assay. The results are expressed as percentages of the control average (i.e., untreated cells). The $\alpha$-syn species generated by $72 \mathrm{~h}$ incubation of preformed $\alpha$-syn fibrils in the presence or absence of GA were added to the cells $48 \mathrm{~h}$ prior to MTT addition (average of 3 wells \pm SD. Statistical analysis was performed using two tailed unpaired $t$-test, *** $p<0.001$; ** $p<0.01$ ).
To assess whether GA disaggregation of $\alpha$-syn fibrils was accompanied by a decrease in $\alpha$-syn toxicity, we then evaluated the toxicity of the $\alpha$-syn species that resulted from the disaggregation experiment on human neuroblastoma M17 cells (Figure 3D). The samples were diluted to final $\alpha$-syn concentrations of $0.5,1$, and $5 \mu \mathrm{M}$. Preformed $\alpha$-syn fibrils incubated for 6 days in the absence of GA decreased cell viability in a dose-dependent manner. However, preformed $\alpha$-syn fibrils incubated with GA for 6 days generated species that were less toxic compared to the control. This trend was most prominent for $5 \mu \mathrm{M} \alpha$-syn, which, when incubated in the absence of GA, induced death of $45 \%$ of the cells, but in the presence of GA, cell death was less than $25 \%$.

\section{THE EFFECT OF GA ON THE SEEDING OF $\alpha$-SYN AGGREGATION}

It has been previously shown that the process of amyloid fibril formation follows a nucleation-dependent polymerization (Jarrett and Lansbury, 1992). According to this model, soluble species generated via the nucleation of oligomeric species (nucleation or lag time phase), which in turn polymerize (polymerization or growth phase) to generate fibrils, reaching thus a final plateau known as the equilibrium phase (Harper et al., 1999). Small aggregates or seeds have been shown to accelerate the nucleation phase of amyloid formation both in vitro and in vivo by a process known as seeding (Jarrett and Lansbury, 1993; Harper and Lansbury, 1997; Volpicelli-Daley et al., 2011; Luk et al., 2012). Given that GA inhibited both $\alpha$-syn fibrillation and disaggregated preformed $\alpha$-syn fibrils, we sought to identify the 
effect of this phenolic acid on the seeding of $\alpha$-syn aggregation. More specifically, mature $\alpha$-syn fibrils were fragmented by sonication to obtain short fibrils, which were employed as "seeds" (Figure 4B). These short fibrillar "seeds" were then added to monomeric $\alpha$-syn, which was allowed to aggregate as described above. As expected, the addition of short fibrillar seeds accelerated the fibrillation of $\alpha$-syn as indicated by the increased Th-S counts. Indeed, with seeding, the extent of $\alpha$-syn fibrillation after $6 \mathrm{~h}$ of incubation was comparable to the fibrillation of the protein incubated for $72 \mathrm{~h}$ without seeding (Figures 1, 4B, respectively).

In order to assess the effect of GA on the seeding of $\alpha$-syn aggregation, 10 and $50 \mu \mathrm{M}$ GA was added to $100 \mu \mathrm{M}$ monomeric $\alpha$-syn containing seeds at a final concentration of $2 \mu \mathrm{M}$, and the mixture was incubated with continuous mixing at $37^{\circ} \mathrm{C}$ for $6 \mathrm{~h}$. GA at $50 \mu \mathrm{M}$ inhibited the seeded fibrillation of $\alpha$-syn by approximately $90 \%$ as indicated by the extremely low Th-S counts. At lower concentrations $(10 \mu \mathrm{M})$, GA also had an inhibitory effect on the seeded fibrillation of $\alpha$-syn but to a smaller extent. At $10 \mu \mathrm{M}, \mathrm{GA}$ inhibited seeded fibrillation by $40-50 \%$ (Figure $4 \mathrm{~A}$ ). These findings were confirmed by TEM (Figure 4B).

\section{THE EFFECT OF GA ON $\alpha$-SYN AGGREGATES-INDUCED TOXICITY}

It has been reported that the oligomeric intermediates are the neurotoxic species in the amyloid fibrillation pathway (Allsop et al., 2001; El-Agnaf et al., 2001, 2006; Vendruscolo et al., 2001; Walsh et al., 2002; Argyriou et al., 2012; Breydo et al., 2012; Colla et al., 2012). As described above, at high concentration (4:1), GA inhibited both early and late aggregate formation, whereas at low concentrations $(2: 1$ and 1:1), it stabilized $\alpha$-syn oligomers (Figure 2A). To determine the effect of GA on toxicity conferred by $\alpha$-syn aggregates, a cell-based toxicity assay, the MTT assay, was conducted with human neuroblastoma M17 cells. The samples were diluted to final $\alpha$-syn concentrations of 5 and $0.5 \mu \mathrm{M}$. The MTT assay showed that $\alpha$-syn aged in the absence of GA decreased cell viability in a dose-dependent manner (Figure 5). However, when $\alpha$-syn was aged in the presence of a high concentration of GA (4:1), there was visible neuroprotection of the cells observed (Figure 5). At low concentrations (2:1 and 1:1), GA exhibited a minor protective effect against $\alpha$-syn toxicity, possibly due to the increase in oligomers as demonstrated by the ELISA results and the immunoblotting analysis (Figures 2A,B, respectively). Our results clearly demonstrate that GA reduced the toxicity of $\alpha$-syn at a high concentration $(100 \mu \mathrm{M})$ by significantly inhibiting the formation of toxic $\alpha$-syn oligomers. The toxicity of the oligomers resulted from $\alpha$-syn: GA 1:4 was compared with $\alpha$-syn oligomers prepared in the absence of GA as described in materials and methods section by MTT assay (Figure 5B). Both samples were characterized for the presence of oligomers by SDS PAGE (Figure 5C) and native PAGE (Figure 5D). It was clear that the oligomers resulting from the incubation of $\alpha$-syn with GA were not toxic comparing with oligomers in absence of GA. Moreover, these oligomers were stable under
A

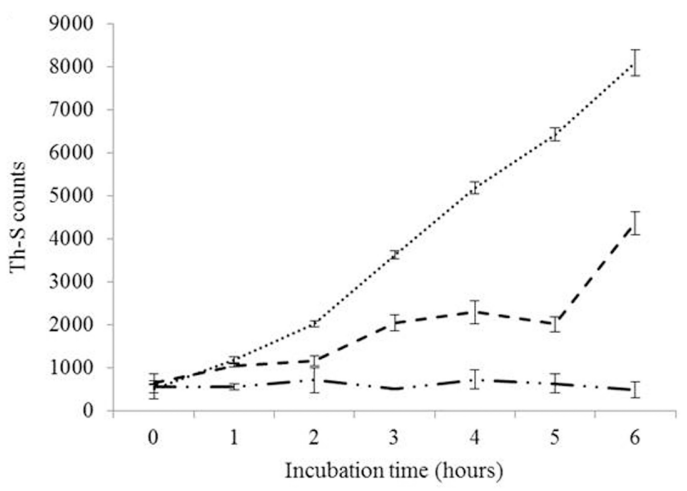

B

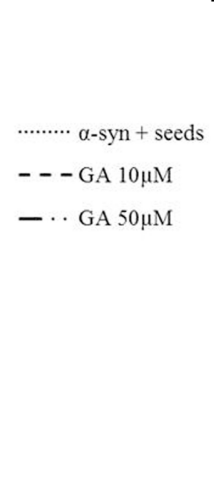

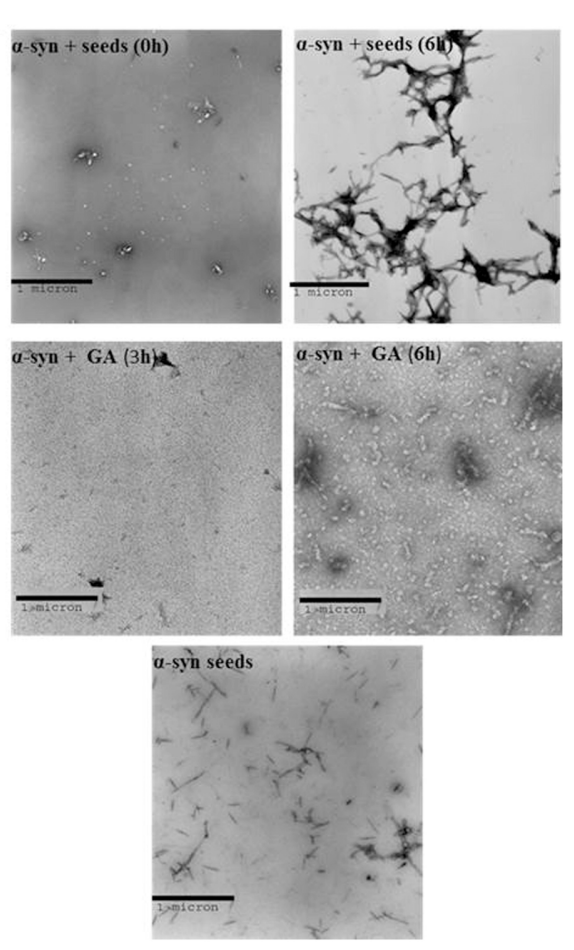

performed in triplicate (average of triplicate measurements \pm standard deviations). (B) Electron microscopy images of negatively stained samples of seeds alone and $\alpha$-syn+ seeds incubated alone or in the presence of GA $(50 \mu \mathrm{M})$ for $6 \mathrm{~h}$ with continuous shaking at $37^{\circ} \mathrm{C}$. Scale bar, $1000 \mathrm{~nm}$. 
A
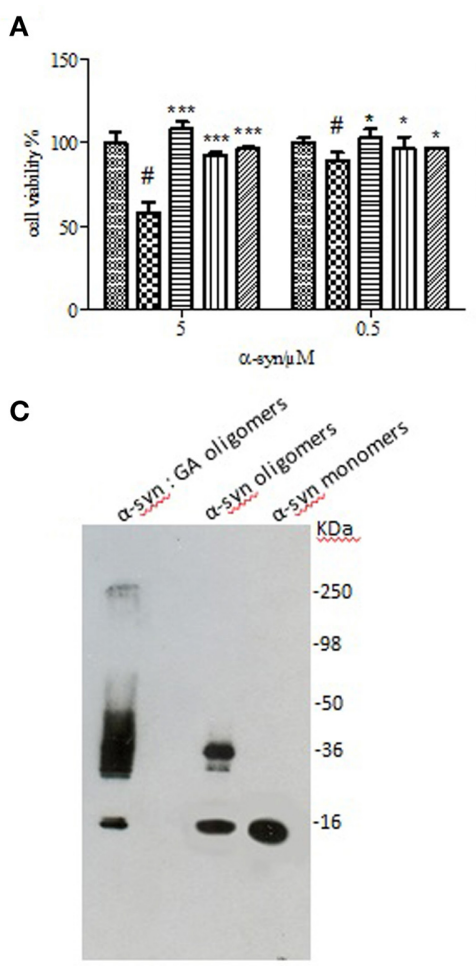

D

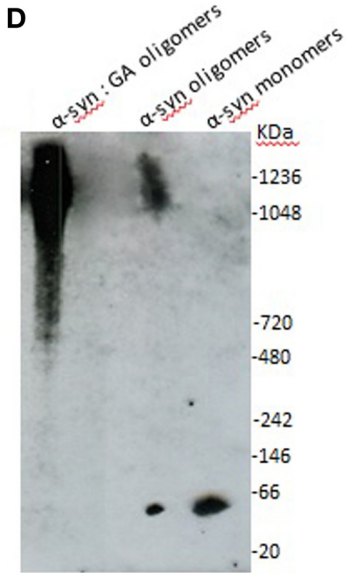

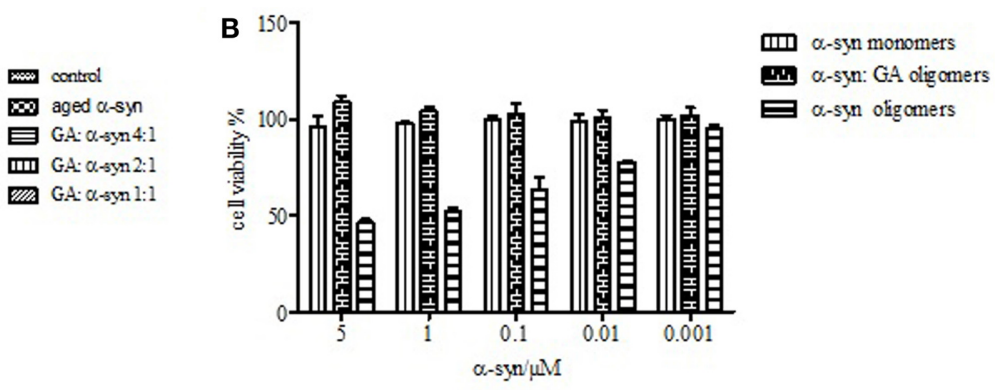

(a)
FIGURE 5 | Effect of GA on the toxicity of aggregated $\alpha$-syn. (A) The viability of BE (2)-M17 human neuroblastoma cells was estimated by the MTT assay. The results are expressed as percentage of the control average (i.e., untreated cells). The cells were treated with aggregated $\alpha$-syn with/without GA for $48 \mathrm{~h}$ prior to MTT addition (average of 3 wells \pm standard deviation. Statistical analysis was performed using two-tailed unpaired $t$-test, $\left.{ }^{* * *}, p<0.001 ;{ }^{*}, p<0.05\right)$. (B) The viability of BE (2)-M17 human neuroblastomacells was estimated by the MTT assay.
The results are expressed as percentage of the control average (i.e., untreated cells). The cells were treated with $\alpha$-syn oligomers in absence or presence of GA for $48 \mathrm{~h}$ prior to MTT addition (average of 3 wells \pm standard deviation. (C) Immunoblot analysis of $\alpha$-syn oligomers generated in presence or absence of GA, separated by electrophoresis in a $15 \%$ SDS-PAGE gel. (D) Immunoblot analysis of $\alpha$-syn oligomers generated in presence or absence of GA, separated by electrophoresis in a $3-12 \%$ Native-PAGE gel. denaturing conditions (Figure 5C) compared those formed in the absence of GA.

\section{GA INHIBITION OF $\alpha$-SYN FIBRILLATION IS MEDIATED VIA BINDING TO THE INTERMEDIATE SPECIES AND FORMING STABLE OLIGOMERS}

The strong inhibitory effect that GA exerted on fibrillation together with the stimulating effect it had on $\alpha$-syn oligomerization at lower GA concentrations, prompted us to investigate further the interaction of GA with $\alpha$-syn oligomers. For this purpose, monomeric $\alpha$-syn $(100 \mu \mathrm{M})$ was aggregated in the presence of GA (GA: $\alpha$-syn 4:1). After 5 days of incubation the samples were centrifuged and the supernatant was injected in a superdex 200 SE column. The elution volume for monomeric $\alpha$-syn was determined by molecular weight standards (Figure $\mathrm{S} 2 \mathrm{~A}$ ), and was eluted in a peak corresponding to column volume of 13-15 mL (Figure S2B), while oligomeric $\alpha$-syn eluted in a peak corresponding to column volume of approximately $7-9 \mathrm{ml}$ (Figure 6A). The fractions corresponding to the oligomeric and monomeric $\alpha$-syn peaks were separately pooled together giving rise to P1 and P2 samples, respectively (Figure 6A), which were concentrated using a speed vac. The $\alpha$-syn species in samples $\mathrm{P} 1$ and P2 were characterized by western blotting (Figure 6B).
According to the western blotting results, the oligomers generated during the incubation of $\alpha$-syn with GA are stable under denaturing conditions (Figures 5C, 6B). Electron microscopy of the same samples indicates the presence of different species of oligomers in P1 (Figure 6D), in agreement with the imunoblotting results (Figure 6B). In order to detect the incorporated GA in the P1 and P2 samples, we exploited the property of GA to produce UV absorbance spectra with two peaks, one at $225 \mathrm{~nm}$ and one at $260 \mathrm{~nm}$. In the sample containing GA: $\alpha-$ syn at 4:1 molar ratio, we detected GA only in the oligomeric P1 samples (Figure 6C). These findings strongly support the hypothesis that GA binds to the oligomeric species and stabilizes them. To further evaluate whether GA interacts with $\alpha$-syn monomers, we monitered a titration of GA into a solution of monomeric $\alpha$-syn using two-dimensional NMR spectroscopy, which provides signals covering the entire amino acid sequence of $\alpha$-syn (Figure 7). At stoichiometries of up to 10:1 GA: $\alpha$-syn we observed no significant chemical shift or resonance intensity changes (Figures S3A-E), confirming that GA does not interact significantly with monomeric $\alpha$-syn. This result is largely in agreement with NMR studies of related compounds Entacapone, Tolcapone and Quercetin (Di Giovanni et al., 2010). 
A

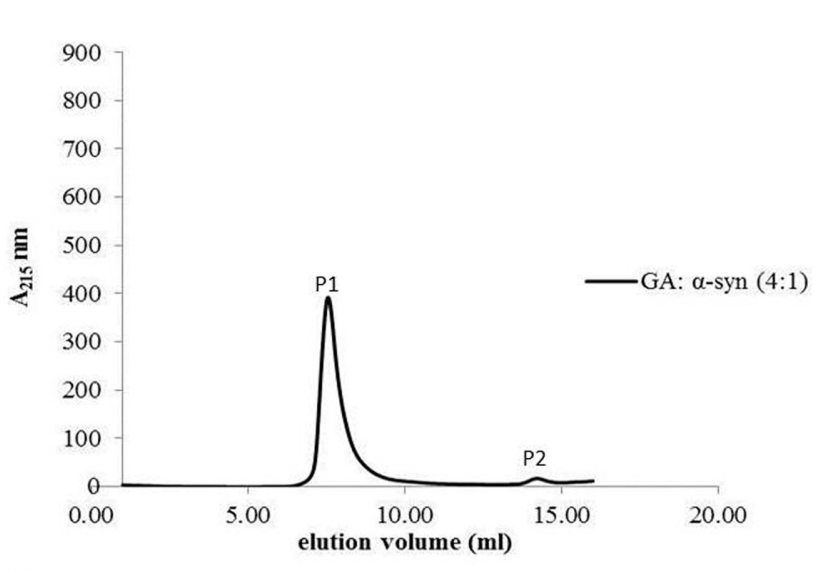

B

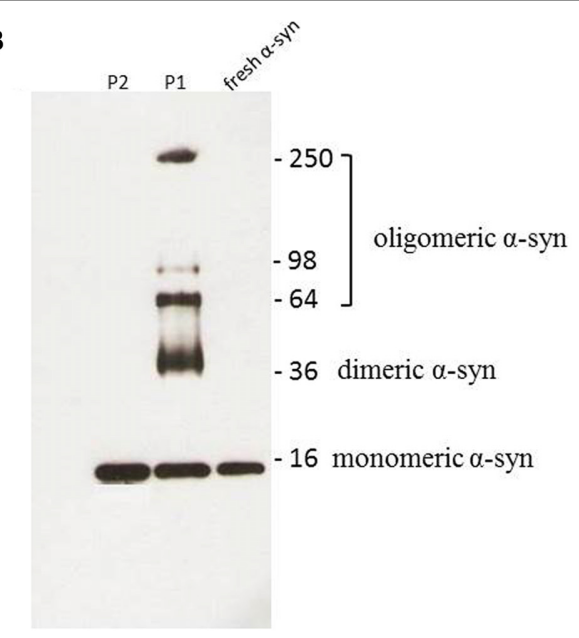

C

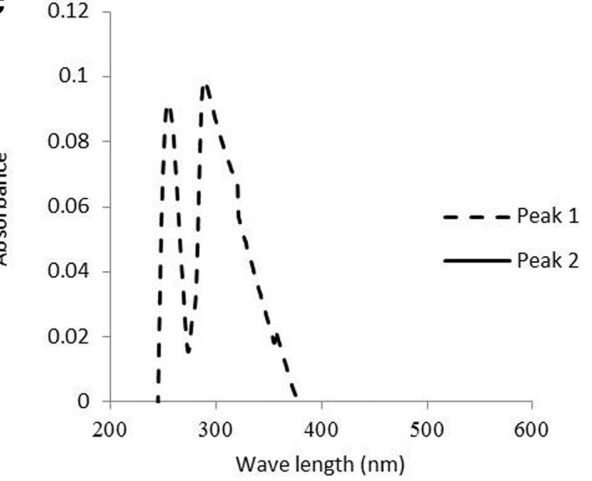

FIGURE 6 | GA binds to $\alpha$-syn oligomers (GA: $\alpha$-syn molar ratio of 4:1). (A) Gel filtration profile of the 5-day-aggregated $\alpha$-syn in the presence of GA at a GA: $\alpha$-syn molar ratio 4:1 ( $\alpha$-syn concentration $=100 \mu \mathrm{M}$ ) using a superdex 200 SE column. P1 sample contains the isolated fractions corresponding to the oligomeric peak and P2 the isolated fractions corresponding to the monomeric peak. The elution was monitored at the absorbance wavelength of $215 \mathrm{~nm}$.

\section{INVESTIGATING THE STRUCTURE-ACTIVITY RELATIONSHIP OF GA INHIBITION OF $\alpha$-SYN FIBRILLATION}

To define the most effective molecular scaffolds against $\alpha$-syn fibril formation and establish a structure-activity relationship for GA, we exploited the structural diversity of benzoic acid derivatives (i.e., hydroxybenzoic acids). Twelve hydroxybenzoic acids, including GA, salicylic acid and gentisic acid (see Table 1) were assessed for their anti-fibrillogenic properties by the Th-S binding assay (Figure 8). The structure of the tested hydroxybenzoic acids is characterized by the presence of a phenyl ring that has a carboxyl group and hydroxyl moieties $(\mathrm{OH})$ attached to the ring at different positions. The selection of the compounds examined in the present study was based on the number (between 0 and 3 ) and position of the hydroxyl groups attached to the phenyl ring (see Table 2).

The kinetics of $\alpha$-syn fibrillation was studied over a period of 5 days in the presence or absence of the phenolic acids. Based on the Th-S assay results (Figure 8 and Table 3), the effect of the twelve phenolic acids on $\alpha$-syn fibrillation varied greatly. The extent of the inhibition of $\alpha$-syn fibrillation was $\sim 99 \%$ for GA, 72\% for 2,4,6-trihydroxybenzoic acid, 60\% for 3,4-dihydroxybenzoic
(B) Immunoblot analysis of the samples P1 and P2 separated by electrophoresis in a 15\% SDS-PAGE gel. (C) UV absorbance spectra of samples $\mathrm{P} 1$ and P2. The UV absorbance was recorded between 200 and $600 \mathrm{~nm}$ employing a $10 \mathrm{~mm}$ quartz cuvette. (D) Electron microscopy images of negatively stained samples P1 and P2 of $\alpha$-syn in the presence of GA (molar ratio of GA: $\alpha$-syn 4:1) purified by SEC. Scale bar, $1000 \mathrm{~nm}$.

acid, $\sim 30 \%$ for five compounds including 2,6-dihydroxybenzoic acid and 4-hydroxybenzoic acid and only 5\% for benzoic acid (see Figure 8 and Table 3). Moreover, three compounds, 2hydroxybenzoic acid (salicylic acid), 3-hydroxybenzoic acid and 3,5-dihydroxybenzoic acid, failed to inhibit $\alpha$-syn fibrillation, with salicylic acid appearing to enhance the aggregation of $\alpha$-syn compared to the control (see Figure 8 and Table 3). By comparing the structures of the most effective compounds and the least effective ones, we conclude that better inhibition of fibrillation is observed when there are a large number of hydroxyl groups $(-\mathrm{OH})$ attached to the phenyl ring of the compound. Moreover, the positions at which the hydroxyl groups are attached to the phenyl ring also appear to play a critical role. GA has hydroxyl groups attached at positions 3 and 5 (see Table 2) and is much more effective than 2,4,6-trihydroxybenzoic acid, which also bears three -OH groups but at different positions (Figure 8). Interestingly, 3,4-dihydroxybenzoic acid, which only lacks an -OH group at position 5 compared to the very effective GA, is the third best inhibitor of $\alpha$-syn fibrillation (Figure 8). Among all the dihydroxybenzoic acids tested, the ones with -OH groups at two consecutive positions, i.e., 3,4-dihydroxybenzoic acid (60\% 


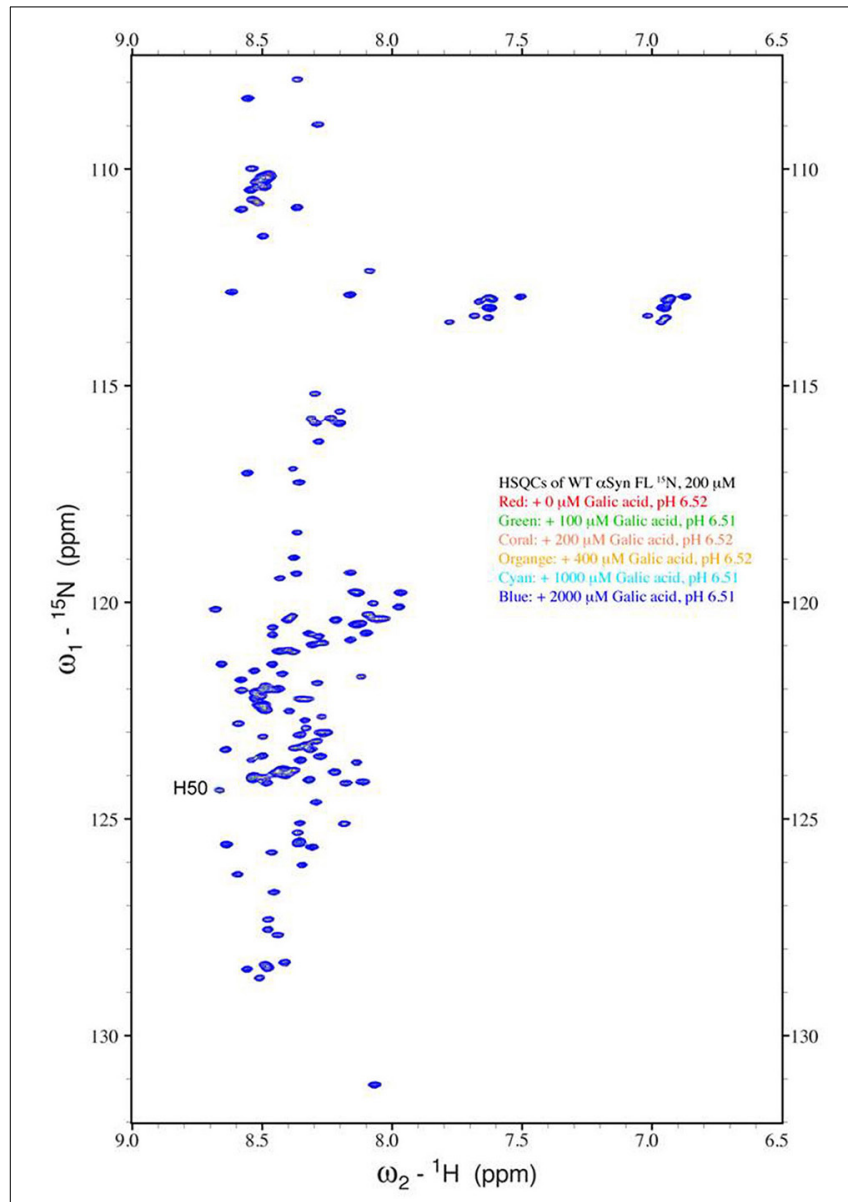

FIGURE 7 | Analysis of GA binding to monomeric $\alpha$-syn by NMR spectroscopy. Proton-Nitrogen correlation (HSOC) spectra of monomeric $\alpha$-syn in the presence of increasing ratios of GA: $\alpha$-syn demonstrating that there are no significant changes in the positions of the NMR resonances, indicating the lack of an interaction between GA and monomeric $\alpha$-syn. Protein concentration was ca. $200 \mu \mathrm{M}$.

inhibition) and 2,3-dihydroxybenzoic acid (44\% inhibition), are more potent inhibitors compared to those that have $-\mathrm{OH}$ groups at non-consecutive positions, i.e., 2,5-dihydroxybenzoic acid (30\% inhibition) and 3,5-dihydroxybenzoic acid (0\% inhibition) (Figure 8). Additionally, all compounds with one $-\mathrm{OH}$ group, as well as the benzoic acid without a $-\mathrm{OH}$ group, failed to show any inhibitory effect on $\alpha$-syn aggregation (Figure 8).

To further investigate the significance of the hydroxyl moieties on the activity of the GA and the other active phenolic acids, three additional compounds with either fluorides or methoxy groups rather than hydroxyl groups attached to their phenyl rings (4-methoxybenzoic acid, 3,4,5-trimethoxybenzoic acid and 3,4,5-trifluorobenzoic acid) (see Table 2) were tested for their ability to inhibit $\alpha$-syn fibril formation. As expected, none of the three tested compounds in any of the three molar ratios tested (compound: $\alpha$-syn 4:1, 2:1, and 1:1) could inhibit $\alpha$-syn fibrillation (Figure 8). This finding provides additional support for the importance of the presence of the hydroxyl group in the phenyl ring.
Table 1 | Description and names of the tested benzoic acid derivatives.

\begin{tabular}{lc}
\hline Name of compound & MW \\
\hline Benzoic acid & 122.12 \\
2-Hydroxybenzoic acid (salicylic acid) & 138.12 \\
3-Hydroxybenzoic acid & 138.12 \\
4-Hydroxybenzoic acid & 138.12 \\
2,3-dihydroxybenzoic acid & 154.12 \\
2,4-dihydroxybenzoic acid & 154.12 \\
2,5-dihydroxybenzoic acid (gentisic acid) & 154.12 \\
2,6-dihydroxybenzoic acid & 154.12 \\
3,4-dihydroxybenzoic acid & 154.12 \\
3,5-dihydroxybenzoic acid & 154.12 \\
2,4,6-trihydroxybenzoic acid & 188.13 \\
3,4,5-trihydroxybenzoic acid (GA) & 170.12 \\
3,4,5-trihfluorobenzoic acid & 176.09 \\
3,4,5-trimethoxybenzoic acid & 212.20 \\
4-methoxybenzoic acid & 152.15 \\
\hline
\end{tabular}

\section{DISCUSSION}

The formation of amyloid aggregates has long been considered responsible for the pathogenesis of several neurodegenerative disorders (Taylor et al., 2002; Dorval and Fraser, 2007; Vekrellis et al., 2011). Although amyloidogenic proteins, such as $\alpha$-syn, are usually found unfolded in their native state (Uversky, 2008; Fauvet et al., 2012), in which they are soluble and non-toxic, they can undergo misfolding resulting in the formation of insoluble aggregates (Seidler et al., 1996; Veldman et al., 1998; Irvine et al., 2008; Kahle, 2008; Stefanis, 2012). These aggregates deposit in various regions of the brain, constituting the main neuropathological feature of several neurodegenerative diseases such as PD, DLB, MSA and approximately the $50 \%$ of Alzheimer's disease cases. In the case of $\alpha$-syn, pathological, biochemical, genetic and animal modeling studies provide compelling evidence that $\alpha$-syn aggregation plays a pivotal role in the pathogenesis of PD and related synucleinopathies. As a consequence, the identification of compounds that can block or reverse the aggregation process of $\alpha$-syn is considered a vital therapeutic strategy against these diseases.

To this end, we assessed the effect of the phenolic compound GA (3,4,5-trihydroxybenzoic acid) on $\alpha$-syn aggregation and toxicity, and we established a structure-activity relationship for GA. By employing an array of biophysical and biochemical techniques, we showed that at a high concentration $(100 \mu \mathrm{M}$, represented by the molar ratio 4:1), GA exerts significant inhibitory effect on both $\alpha$-syn fibrillation (Figures 1A,B) and oligomerization (Figures 2A,B), as well as seeded fibrillation of $\alpha$-syn (Figure 4A). In accordance with these findings, TEM images revealed that $\alpha$ syn in the presence of GA at high concentration only formed a few short, thin fibrils that had a fragmented appearance (Figure 1D) accounting for the low Th-S counts (Figure 1A) and the minor wavelength shift in the CR binding assay (Figure 1B). At lower GA concentrations (especially at 1:1 molar ratio), however, the ability of the GA to inhibit $\alpha$-syn fibrillation was less dramatic and accompanied by a striking increase in the oligomeric content (Figure 2A), indicating GA may stabilize the oligomeric structure. 


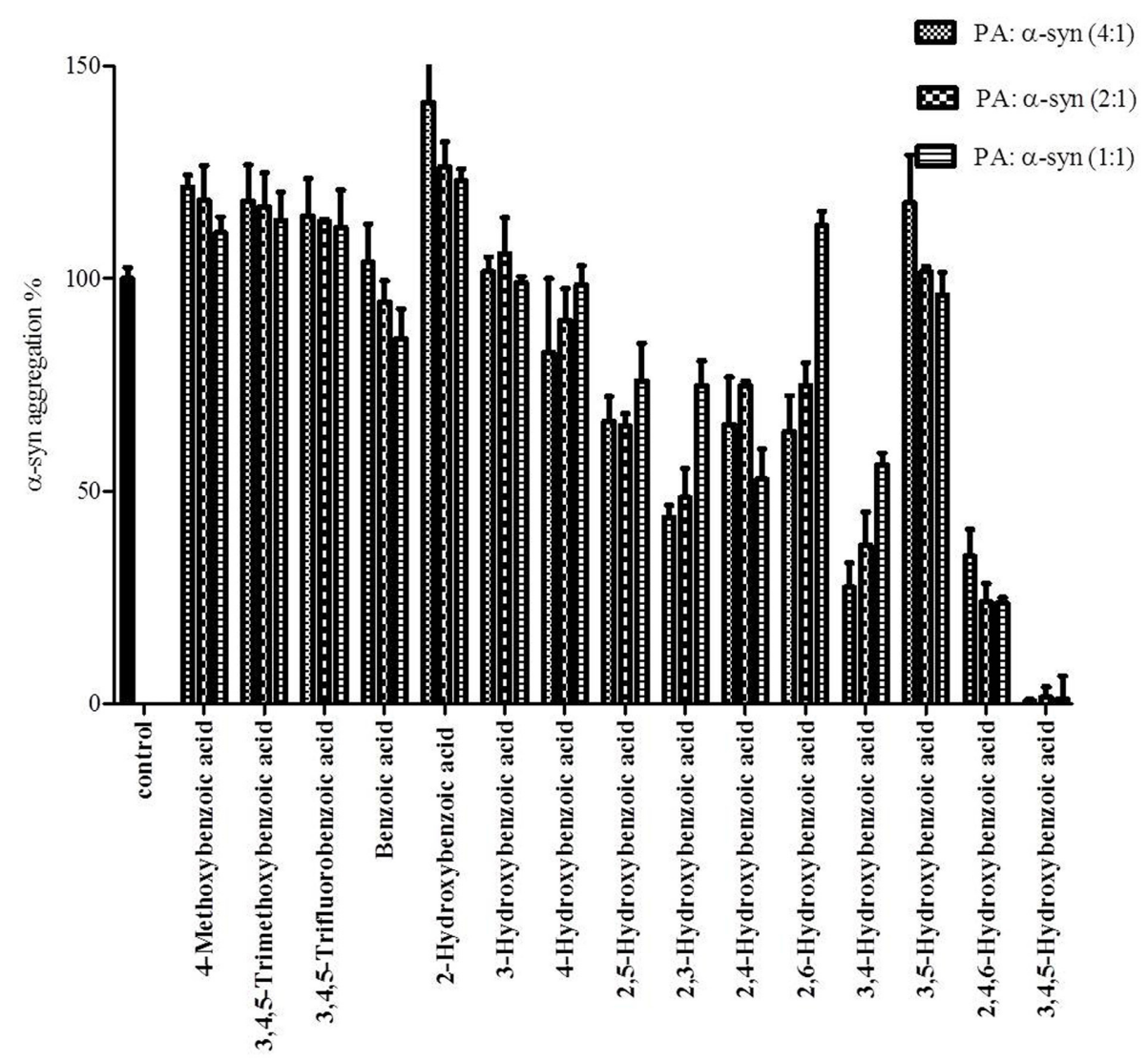

FIGURE 8 | Effect of different benzoic acid derivatives (phenolic acids, PA) and the effect of methoxy and fluoro groups in benzoic acid derivatives on $\alpha$-syn fibrillation. Samples of $\alpha$-syn $(25 \mu \mathrm{M})$ were incubated alone or in the presence of different benzoic acid derivatives (PA: $\alpha$-syn molar ratios of
$4: 1,2: 1,1: 1)$ for 6 days with continuous shaking at $37^{\circ} \mathrm{C}$. The fibril formation was measured by the Th-S binding assay and expressed as a percentage of the fibril content of $\alpha$-syn aged alone. The assay was performed in triplicate (average of triplicate measurements \pm standard deviations).
Size exclusion chromatography combined with UV spectroscopy for detection of the incorporated GA in $\alpha$-syn confirmed that GA binds to $\alpha$-syn oligomers (Figure 6C). Furthermore, GA was able to disaggregate preformed $\alpha$-syn fibrils (Figures 3A-C), generating species that possessed a decreased $\beta$-sheet content (Figure 3B) and were less toxic to the cells (Figure 3D). Previous studies have also shown that the flavonoid baicalein, as well as other antioxidant compounds inhibit $\alpha$-syn fibrillation and disaggregate preaggregated fibrils (Zhu et al., 2004; Ono and Yamada, 2006). GA was also shown to alleviate $\alpha$-syn aggregates associated-toxicity in neuroblastoma M17 cells (Figures 5A,B).

These results are in agreement with previous studies indicating that GA possesses anti-fibrillation, oligomer-stabilizing and neuroprotective properties against $\alpha$-syn and $A \beta$ cytotoxicity (Di Giovanni et al., 2010). Indeed, GA was shown to stabilize biotinylated $A \beta_{1-42}$ oligomers (Levine et al., 2012) and to inhibit the in vitro conversion of low molecular weight $\mathrm{A} \beta_{1-42}$ protofibrils into fibrils by 50\% (Di Giovanni et al., 2010). Additionally, GA was reported to block the fibrillation of $\alpha$-syn in vitro and to suppress the ability of short $\alpha$-syn fibrils to seed the aggregation of monomeric $\alpha$-syn (Di Giovanni et al., 2010). Furthermore, GA exerts a neuroprotective effect on cells against the cytotoxicity of $\alpha$-syn and $A \beta_{1-42}$ (Di Giovanni et al., 2010), possibly due to the inhibition of the amyloid aggregation but potentially also due to the anti-inflammatory (Kim et al., 2011), anti-oxidant (Ban et al., 2008; Hong et al., 2012) and anti-apoptotic properties it possesses (Hong et al., 2012).

Based on our findings using both SEC and NMR, as well as previous reports, GA inhibits $\alpha$-syn fibrillation not by interacting with the monomeric $\alpha$-syn - similar to many other phenolic compounds (reviewed in Porat et al., 2006)-, and preventing it from polymerizing, but rather by stabilizing the structure of oligomeric $\alpha$-syn (Figures 2B, 6C) which appears to be non-toxic (Figure 5). These findings are in accordance with previous studies indicating that polyphenolic compounds, such as baicalein, curcumin and epigallocatechin gallate (EGCG) induce the formation of soluble, non-toxic oligomers (Zhu et al., 2004; Masuda et al., 2006). From a therapeutic point of view, this represents a major advantage, as GA may not interfere with the physiological function of the monomeric $\alpha$-syn (Wagner et al., 2013). It has been reported that phenolic compounds such as curcumin inhibit $A \beta$ aggregation due to conformation-dependent binding (Yang et al., 2005). Consistent with this model of inhibition, GA also inhibited the seeding ability of short fibrillar $\alpha$-syn. Although 
Table 2 | The compounds were divided into four groups based on the number of hydroxyl groups attached to the phenyl ring.

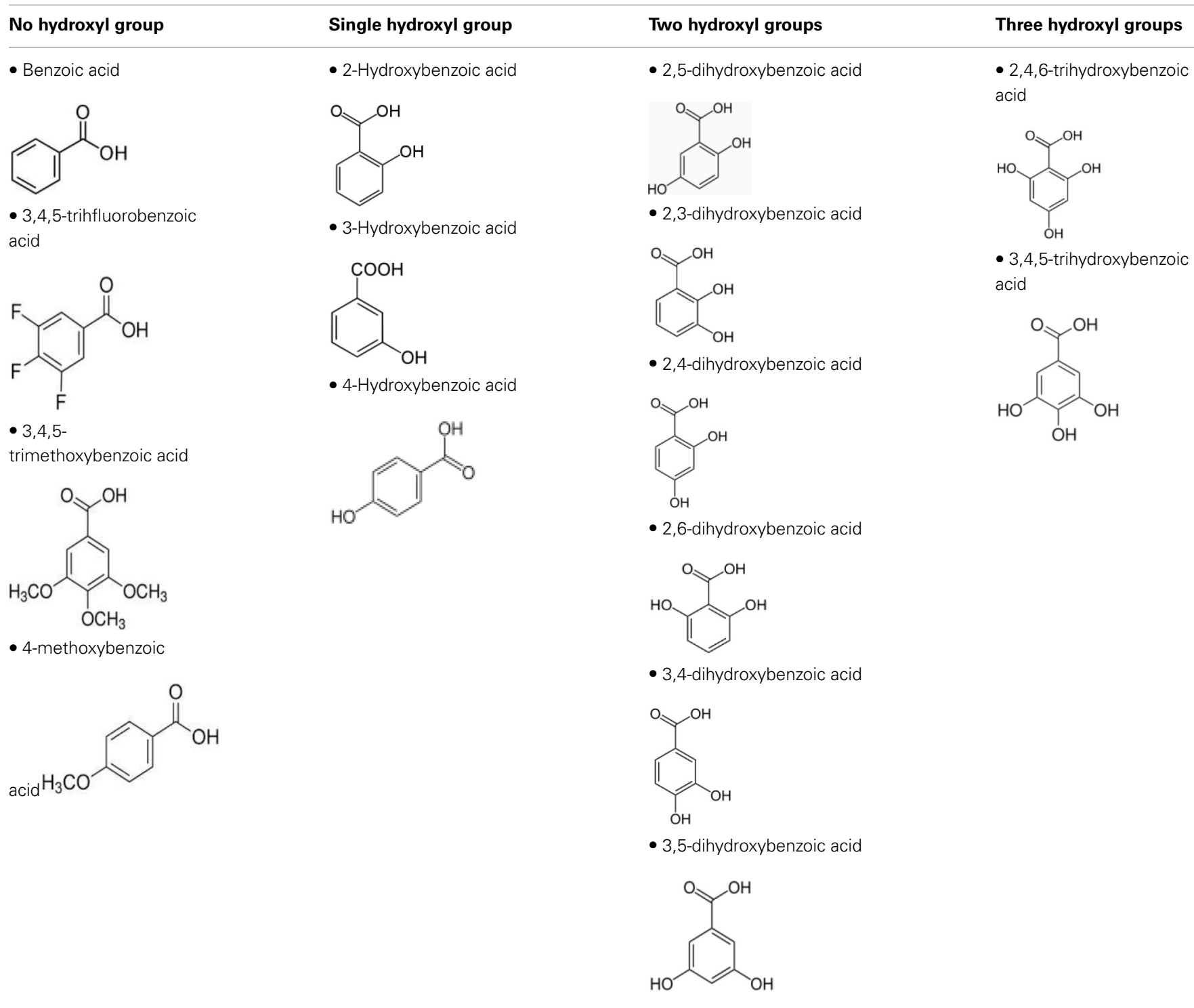

the presence of oligomers can be transient as they assemble into fibrils (Kaylor et al., 2005; Fink, 2006), there are studies revealing that in the presence of the polyphenolic compound EGCG, they can shift off-pathway from fibrillation (Ehrnhoefer et al., 2008). Indeed, EGCG was shown to promote the formation of less toxic oligomers that were exceedingly stable and incapable of contributing to the aggregation process (Ehrnhoefer et al., 2008). As it is also a phenolic compound, GA may interact with $\alpha$-syn oligomers by a mechanism similar to that of EGCG, accelerating the formation of off-pathway oligomers and leading to the accumulation of non-toxic oligomers and the interruption of the fibrillation process.

To gain insight into the mechanism of action of GA and to establish a structure-function relationship for this phenolic acid, we evaluated the effect of various structurally similar phenolic acids on $\alpha$-syn fibrillation. The results generated from this approach point toward a structure-related effect on $\alpha$-syn fibrillation in which the better inhibitors were observed to have a greater number of $-\mathrm{OH}$ groups attached to the phenyl ring that were directly conjugated to the carboxylic acid arm (see Figure 8). In fact, the ranking of the anti-fibrillogenic potency of all the tested phenolic compounds could be represented as follows: trihydroxybenzoic acid > dihydroxybenzoic acid > monohydroxybenzoic acid = benzoic acid. This finding is in accordance with previous studies indicating that the potency of certain polyphenolic compounds to inhibit and disaggregate $\alpha$ syn oligomers correlates with the number of vicinal -OH groups present on a single phenyl ring (Masuda et al., 2006; Caruana et al., 2011). The significance of the presence of the hydroxyl group on the phenyl ring was further stressed by the fact that the compounds that possess appendages other than hydroxyl groups on the phenyl rings failed to inhibit $\alpha$-syn aggregation. 


\begin{tabular}{|c|c|c|c|}
\hline Symbol & Compound & $\mathrm{OH}$ group & $\begin{array}{c}\text { Inhibition } \% \text { of } \\
\alpha \text {-syn aggregation }\end{array}$ \\
\hline PA1 & 3,4,5-trihydroxybenzoic acid & 3 & 99 \\
\hline PA2 & 2,4,6-trihydroxybenzoic acid & 3 & 72 \\
\hline PA3 & 2,3-dihydroxybenzoic acid & 2 & 44 \\
\hline PA4 & 2,4-dihydroxybenzoic acid & 2 & 35 \\
\hline PA5 & 2,5-dihydroxybenzoic acid & 2 & 30 \\
\hline PA6 & 2,6-dihydroxybenzoic acid & 2 & 16 \\
\hline PA7 & 3,4-dihydroxybenzoic acid & 2 & 60 \\
\hline PA8 & 3,5-dihydroxybenzoic acid & 2 & 0 \\
\hline PA9 & 2-hydroxybenzoic acid & 1 & 0 \\
\hline PA10 & 3-Hydroxybenzoic acid & 1 & 0 \\
\hline PA11 & 4-Hydroxybenzoic acid & 1 & 9 \\
\hline PA12 & Benzoic acid & 0 & 5 \\
\hline PA13 & 4-methoxybenzoic acid & 0 & 0 \\
\hline PA14 & 3,4,5-trimethoxybenzoic acid & 0 & 0 \\
\hline PA15 & 3,4,5-trifluorobenzoic acid & 0 & 0 \\
\hline
\end{tabular}

At this point, it is more than an idle speculation to suggest that the inhibition capacity of the phenolic compounds tested is not only dependent on the number of hydroxyl moieties but also on their position and conjugation with respect to the benzoic acid appendage. Comparison of the two trihydroxybenzoic acids tested in this study, GA (3,4,5-trihydroxybenzoic acid) and 2,4,6-trihydroxybenzoic acid, indicates that although both compounds have a total of three -OH groups, the former is a much more potent inhibitor of $\alpha$-syn fibrillation compared to the latter, strongly suggesting that it is not only the total number of -OH groups present in the molecule but also their position that is important. Additionally, comparison of the dihydroxybenzoic acids employed in the present study reveals that the presence of -OH groups in two consecutive positions and conjugated with the carboxyl group [e.g., 3,4-dihydroxybenzoic acid (60\% inhibition) and 2,3-dihydroxybenzoic acid (44\% inhibition)] renders the compounds more active compared to those that have hydroxyl groups at non-consecutive positions (e.g., 2,5-dihydroxybenzoic acid (30\% inhibition) and 3,5-dihydroxybenzoic acid (0\% inhibition) (see Figure 8 and Table 3 ). The importance of the $4-\mathrm{OH}$ moiety is emphasized by the fact that of the three hydroxybenzoic acids tested, only 4-hydroxybenzoic acid could partially inhibit $\alpha$-syn fibrillation. Among the dihydroxybenzoic acids, 3,4-dihydroxybenzoic acid was the most potent of all, inhibiting fibrillation by $60 \%$ (Table 3 ). Taken as a whole, these data show that the total number of $-\mathrm{OH}$ groups present in the molecule is important for the compound activity and that the position of the overall -OH groups also affects activity, with a hydroxyl group at position 4 appearing to play a significant role. Thus, the presence of three vicinal hydroxyl groups, as in 3,4,5-trihydroxy benzoic acid (GA), or the three homo-vicinal groups, as in 2,4,6trihydroxy benzoic acid, with one $-\mathrm{OH}$ group at position 4, renders the compounds able to inhibit $\alpha$-syn fibrillation to a great extent.
Given that all the compounds tested in this study have a phenyl ring in their structures, the phenyl structure itself is clearly not sufficient to inhibit $\alpha$-syn aggregation. Consequently, the inhibition ability of such compounds must be achieved by the presence of additional - $\mathrm{OH}$ groups attached to the phenyl ring. The conjugated hydroxyl groups with the carboxylic acid appendage can easily form quinone structures (see Figure S4). It is worth mentioning that quinone formation has been previously reported as the potential mechanism of inhibition of $\alpha$-syn fibrillation by the phenolic compound baicalein (Zhu et al., 2004). The formation of such oxidized derivatives can increase the stability of the compound binding to the $\alpha$-syn fibrils (Taniguchi et al., 2005), possibly accounting for the increased inhibitory potency of the compounds with more $-\mathrm{OH}$ groups present in their structures. Quinones are likely to interact with the aromatic residues of $\alpha$-syn, interfering with their $\pi-\pi$ stacking (Porat et al., 2006; Ebrahimi and Schluesener, 2012; Hamley, 2012). Moreover, quinones have a planar orientation that allows them to align with the hydrophobic groove of $\alpha$-syn fibrils, which possess an in-register organization of side chains in the regular cross- $\beta$-sheet structure (Porat et al., 2006; Ebrahimi and Schluesener, 2012; Hamley, 2012). Therefore, the inhibition of $\alpha$-syn fibrillation by GA and 2,4,6-trihydroxybenzoic acid could be attributed to the combined properties of their three $-\mathrm{OH}$ groups and quinone-forming structures. Based on these results, we propose a plausible model to interpret the inhibitory and disassembling effects of GA. Two dominant ligand-protein interactions contribute to inhibitor potency, (a) the dipole-dipole interactions of the quinone and (b) the dipole-dipole interactions of the diquinone group. The most potent inhibitors, with 3,4,5-trihydroxy and 2,4,6-trihydroxy substitution patterns (GA and 2,4,6-trihydroxy benzoic acid, respectively), (see Figure S4c), enable both hydrophobic and dipole-dipole interactions. The $\pi$ $\pi$ stacking is known to play a role in the binding affinity of inhibitors to aggregates, increasing the potential for quinone formation of each compound generally leading to increased binding affinity and, therefore, increased inhibitor potency. Consistent with this interpretation, ortho- and meta-hydroxybenzoic acid, as well as benzoic acid (see Figure S4f) showed no inhibition effect compared to their para- and ortho-paradihydroxy (Figure S4a) counterparts due to the inability of the former to form quinone-like ligands (Figures S4f, d).

Although monocyclic compounds such as phenolic acids were previously shown to be very poor inhibitors of aggregation (Reinke and Gestwicki, 2007), later studies revealed that monocyclic compounds could suppress amyloid assembly by interacting with oligomers, stabilizing their structure and conformation, thus inhibiting the transition to $\beta$-sheet structures (Levy-Sakin et al., 2009). It is therefore likely that phenolic acids, especially GA, act in a similar way, interacting with oligomers and stabilizing their structure through the $-\mathrm{OH}$ groups, preventing the transition to $\beta$-sheet-rich structures. This may explain the increased oligomeric content detected at low GA concentrations and the low $\beta$-sheet content of $\alpha$-syn species generated in the presence of GA.

In summary, we conducted a systematic study of the effect of GA on $\alpha$-syn fibrillation, oligomerization and toxicity, and we concluded that GA inhibits $\alpha$-syn fibrillation and toxicity by 
stabilizing the non-toxic oligomeric structure of $\alpha$-syn through oligomer binding. Our study points toward a structure-related inhibition, with the number of $-\mathrm{OH}$ moieties and their position around the phenyl ring playing a fundamental role. Based on the above experimental results, we propose a model to interpret the inhibitory and disassembling effects of GA derivatives on $\alpha$ syn aggregation (see Figure S5). $\alpha$-Syn aggregation is a sequential process in which unstructured $\alpha$-syn monomers undergo conformational transition and re-ordering to form oligomers and then finally amyloid fibrils (routes $\mathrm{a} \rightarrow \mathrm{b} \rightarrow \mathrm{c} \rightarrow \mathrm{d}$, Figure S5). The analysis of the results obtained from this study shows that GA derivatives can prolong the nucleation process, suggesting that these scaffolds can bind to unstructured $\alpha$-syn oligomers and prevent protein association (Figure S5, route $\mathrm{a} \rightarrow \mathrm{f}$ ) and/or slow down the conformational transition to structured oligomers, the precursors of amyloid fibrils. GA derivatives interact with $\alpha$ syn monomers and/or oligomers through $\pi-\pi$ stacking, leading to the formation of an energy barrier that prevents the association of $\alpha$-syn molecules. Meanwhile, GA derivatives through their respective quinone oxidation products could induce structural disruption to the local $\beta$-sheet of $\alpha$-syn fibrils via strong binding to the $\beta$-sheet groove regions of $\alpha$-syn fibrils, leading to fibril disaggregation $(\mathrm{d} \rightarrow \mathrm{e})$. It should be noted that, due to the hydrophobic aromatic nature and planar structure of quinones, they might interact with $\alpha$-syn fibrils by relatively nonspecific dipole-dipole interactions with $\beta$-sheet-rich side chains. This binding mode implies that quinones could have a general inhibitory effect on the aggregation of $\alpha$-syn.

\section{ACKNOWLEDGMENTS}

We thank Hyangshuk Rhim (The catholic University College of Medicine,Seoul, Korea) for kindly providing us the pGEX4 T1- $\alpha$-syn plasmid. This work is supported by grant from Sheikh Hamdan Bin Rashid Al Maktoum Award for Medical Sciences (Dubai, UAE; Grant MRG-23/2005-2006), and College of Medicine and Health Sciences (Al Ain, UAE; Interdisciplinary Research Project \# 01-03-8-12/08). David Eliezer is supported by NIH/NIA grant AG019391. Mustafa T. Ardah was supported by United Arab Emirates University- PhD scholarship. This publication was made possible by NPRP grant \# 4-1371-1-223 from the Qatar National Research Fund (a member of Qatar Foundation). The statements made herein are solely the responsibility of the author $[s]$.

\section{SUPPLEMENTARY MATERIAL}

The Supplementary Material for this article can be found online at: http://www.frontiersin.org/journal/10.3389/fnagi. 2014.00197/abstract

\section{REFERENCES}

Allsop, D., Swanson, L., Moore, S., Davies, Y., York, A., El-Agnaf, O. M., et al. (2001). Fluorescence anisotropy: a method for early detection of Alzheimer beta-peptide (Abeta) aggregation. Biochem. Biophys. Res. Commun. 285, 58-63. doi: 10.1006/bbrc.2001.5123

Appel-Cresswell, S., Vilarino-Guell, C., Encarnacion, M., Sherman, H., Yu, I., Shah, B., et al. (2013). Alpha-synuclein p.H50Q, a novel pathogenic mutation for Parkinson's disease. Mov. Disord. 28, 811-813. doi: 10.1002/mds. 25421
Argyriou, A., Dermentzaki, G., Papasilekas, T., Moraitou, M., Stamboulis, E., Vekrellis, K., et al. (2012). Increased dimerization of alpha-synuclein in erythrocytes in Gaucher disease and aging. Neurosci. Lett. 528, 205-209. doi: 10.1016/j. neulet.2012.08.069

Ban, J. Y., Nguyen, H. T., Lee, H. J., Cho, S. O., Ju, H. S., Kim, J. Y., et al. (2008). Neuroprotective properties of gallic acid from Sanguisorbae radix on amyloid beta protein (25-35)-induced toxicity in cultured rat cortical neurons. Biol. Pharm. Bull. 31, 149-153. doi: 10.1248/bpb.31.149

Bastianetto, S., Yao, Z. X., Papadopoulos, V., and Quirion, R. (2006). Neuroprotective effects of green and black teas and their catechin gallate esters against beta-amyloid-induced toxicity. Eur. J. Neurosci. 23, 55-64. doi: 10.1111/ j.1460-9568.2005.04532.x

Breydo, L., Wu, J. W., and Uversky, V. N. (2012). A-synuclein misfolding and Parkinson's disease. Biochim. Biophys. Acta 1822, 261-285. doi: 10.1016/j.bbadis. 2011.10.002

Bussell, R., and Eliezer, D. (2003). A structural and functional role for 11-mer repeats in alpha-synuclein and other exchangeable lipid binding proteins. J. Mol. Biol. 329, 763-778. doi: 10.1016/S0022-2836(03)00520-5

Camilleri, A., and Vassallo, N. (2014). The centrality of mitochondria in the pathogenesis and treatment of Parkinson's Disease. CNS Neurosci. Ther. 20, 591-602. doi: $10.1111 / \mathrm{cns} .12264$

Cannon, J. R., Geghman, K. D., Tapias, V., Sew, T., Dail, M. K., Li, C., et al. (2013). Expression of human E46K-mutated $\alpha$-synuclein in BAC-transgenic rats replicates early-stage Parkinson's disease features and enhances vulnerability to mitochondrial impairment. Exp. Neurol. 240, 44-56. doi: 10.1016/j.expneurol. 2012.11.007

Caruana, M., Högen, T., Levin, J., Hillmer, A., Giese, A., and Vassallo, N. (2011). Inhibition and disaggregation of $\alpha$-synuclein oligomers by natural polyphenolic compounds. FEBS Lett. 585, 1113-1120. doi: 10.1016/j.febslet.2011.03.046

Colla, E., Jensen, P. H., Pletnikova, O., Troncoso, J. C., Glabe, C., and Lee, M. K. (2012). Accumulation of toxic $\alpha$-synuclein oligomer within endoplasmic reticulum occurs in $\alpha$-synucleinopathy in vivo. J. Neurosci. 32, 3301-3305. doi: 10.1523/JNEUROSCI.5368-11.2012

Conway, K. A., Harper, J. D., and Lansbury, P. T. (2000). Fibrils formed in vitro from alpha-synuclein and two mutant forms linked to Parkinson's disease are typical amyloid. Biochemistry 39, 2552-2563. doi: 10.1021/bi991447r

Di Giovanni, S., Eleuteri, S., Paleologou, K. E., Yin, G., Zweckstetter, M., Carrupt, P. A., et al. (2010). Entacapone and tolcapone, two catechol O-methyltransferase inhibitors, block fibril formation of alpha-synuclein and beta-amyloid and protect against amyloid-induced toxicity. J. Biol. Chem. 285, 14941-14954. doi: 10.1074/jbc.M109.080390

Dorval, V., and Fraser, P. E. (2007). SUMO on the road to neurodegeneration. Biochim. Biophys. Acta 1773, 694-706. doi: 10.1016/j.bbamcr.2007.03.017

Ebrahimi, A., and Schluesener, H. (2012). Natural polyphenols against neurodegenerative disorders: potentials and pitfalls. Ageing Res. Rev. 11, 329-345. doi: 10.1016/j.arr.2012.01.006

Ehrnhoefer, D. E., Bieschke, J., Boeddrich, A., Herbst, M., Masino, L., Lurz, R., et al. (2008). EGCG redirects amyloidogenic polypeptides into unstructured, off-pathway oligomers. Nat. Struct. Mol. Biol. 15, 558-566. doi: 10.1038/nsmb. 1437

El-Agnaf, O. M., Jakes, R., Curran, M. D., and Wallace, A. (1998). Effects of the mutations Ala30 to Pro and Ala53 to Thr on the physical and morphological properties of alpha-synuclein protein implicated in Parkinson's disease. FEBS Lett. 440, 67-70. doi: 10.1016/S0014-5793(98)01419-7

El-Agnaf, O. M., Nagala, S., Patel, B. P., and Austen, B. M. (2001). Non-fibrillar oligomeric species of the amyloid ABri peptide, implicated in familial British dementia, are more potent at inducing apoptotic cell death than protofibrils or mature fibrils. J. Mol. Biol. 310, 157-168. doi: 10.1006/jmbi.2001.4743

El-Agnaf, O. M., Salem, S. A., Paleologou, K. E., Curran, M. D., Gibson, M. J., Court, J. A., et al. (2006). Detection of oligomeric forms of alpha-synuclein protein in human plasma as a potential biomarker for Parkinson's disease. FASEB J. U.S.A. 20, 419-425. doi: 10.1096/fj.03-1449com

Eliezer, D., Kutluay, E., Bussell, R., and Browne, G. (2001). Conformational properties of alpha-synuclein in its free and lipid-associated states. J. Mol. Biol. 307, 1061-1073. doi: 10.1006/jmbi.2001.4538

Fauvet, B., Mbefo, M. K., Fares, M. B., Desobry, C., Michael, S., Ardah, M. T., et al. (2012). $\alpha$-Synuclein in central nervous system and from erythrocytes, mammalian cells, and Escherichia coli exists predominantly as disordered monomer. J. Biol. Chem. 287, 15345-15364. doi: 10.1074/jbc.M111.318949 
Feany, M. B., and Bender, W. W. (2000). A Drosophila model of Parkinson's disease. Nature 404, 394-398. doi: 10.1038/35006074

Fink, A. L. (2006). The aggregation and fibrillation of alpha-synuclein. Acc. Chem. Res. 39, 628-634. doi: 10.1021/ar050073t

Giasson, B. I., Uryu, K., Trojanowski, J. Q., and Lee, V. M. (1999). Mutant and wild type human alpha-synucleins assemble into elongated filaments with distinct morphologies in vitro. J. Biol. Chem. 274, 7619-7622. doi: $10.1074 / \mathrm{jbc} .274 .12 .7619$

Greenbaum, E. A., Graves, C. L., Mishizen-Eberz, A. J., Lupoli, M. A., Lynch, D. R., Englander, S. W., et al. (2005). The E46K mutation in alpha-synuclein increases amyloid fibril formation. J. Biol. Chem. 280, 7800-7807. doi: 10.1074/jbc.M411638200

Hamley, I. W. (2012). The amyloid beta peptide: a chemist's perspective. Role in Alzheimer's and fibrillization. Chem. Rev. 112, 5147-5192. doi: $10.1021 / \mathrm{cr} 3000994$

Harper, J. D., and Lansbury, P. T. (1997). Models of amyloid seeding in Alzheimer's disease and scrapie: mechanistic truths and physiological consequences of the time-dependent solubility of amyloid proteins. Annu. Rev. Biochem. 66, 385-407. doi: 10.1146/annurev.biochem.66.1.385

Harper, J. D., Wong, S. S., Lieber, C. M., and Lansbury, P. T. (1999). Assembly of A beta amyloid protofibrils: an in vitro model for a possible early event in Alzheimer's disease. Biochemistry 38, 8972-8980. doi: 10.1021/bi9904149

Hashimoto, M., Hsu, L. J., Sisk, A., Xia, Y., Takeda, A., Sundsmo, M., et al. (1998). Human recombinant NACP/alpha-synuclein is aggregated and fibrillated in vitro: relevance for Lewy body disease. Brain Res. 799, 301-306. doi: 10.1016/S0006-8993(98)00514-9

Ho, H. H., Chang, C. S., Ho, W. C., Liao, S. Y., Lin, W. L., and Wang, C. J. (2013). Gallic acid inhibits gastric cancer cells metastasis and invasive growth via increased expression of RhoB, downregulation of AKT/small GTPase signals and inhibition of NF-кB activity. Toxicol. Appl. Pharmacol. 266, 76-85. doi: 10.1016/j.taap.2012.10.019

Ho, J. H., and Hong, C. Y. (2011). Salvianolic acids: small compounds with multiple mechanisms for cardiovascular protection. J. Biomed. Sci. 18, 30. doi: 10.1186/1423-0127-18-30

Hong, S. Y., Jeong, W. S., and Jun, M. (2012). Protective effects of the key compounds isolated from Corni fructus against $\beta$-amyloid-induced neurotoxicity in PC12 cells. Molecules 17, 10831-10845. doi: 10.3390/molecules170910831

Ibáñez, P., Bonnet, A. M., Débarges, B., Lohmann, E., Tison, F., Pollak, P., et al. (2004). Causal relation between alpha-synuclein gene duplication and familial Parkinson's disease. Lancet 364, 1169-1171. doi: 10.1016/S0140-6736(04) 17104-3

Imai, Y., Venderova, K., Park, D. S., Cai, H., and Schmidt, E. (2011). Animal models of Parkinson's disease. Parkinsons Dis. 2011, 364328. doi: 10.4061/2011/364328

Irvine, G. B., El-Agnaf, O. M., Shankar, G. M., and Walsh, D. M. (2008). Protein aggregation in the brain: the molecular basis for Alzheimer's and Parkinson's diseases. Mol. Med. 14, 451-464. doi: 10.2119/2007-00100.Irvine

Jarrett, J. T., and Lansbury, P. T. (1992). Amyloid fibril formation requires a chemically discriminating nucleation event: studies of an amyloidogenic sequence from the bacterial protein OsmB. Biochemistry 31, 12345-12352. doi: 10.1021/bi00164a008

Jarrett, J. T., and Lansbury, P. T. (1993). Seeding "one-dimensional crystallization" of amyloid: a pathogenic mechanism in Alzheimer's disease and scrapie? Cell 73, 1055-1058.

Junn, E., and Mouradian, M. M. (2002). Human alpha-synuclein over-expression increases intracellular reactive oxygen species levels and susceptibility to dopamine. Neurosci. Lett. 320, 146-150. doi: 10.1016/S0304-3940(02)00016-2

Kahle, P. J. (2008). alpha-Synucleinopathy models and human neuropathology: similarities and differences. Acta Neuropathol. 115, 87-95. doi: 10.1007/s00401007-0302-x

Kahle, P. J., Neumann, M., Ozmen, L., Müller, V., Odoy, S., Okamoto, N., et al. (2001). Selective insolubility of alpha-synuclein in human Lewy body diseases is recapitulated in a transgenic mouse model. Am. J. Pathol. 159, 2215-2225. doi: $10.1016 /$ S0002-9440(10)63072-6

Kaylor, J., Bodner, N., Edridge, S., Yamin, G., Hong, D. P., and Fink, A. L. (2005). Characterization of oligomeric intermediates in alpha-synuclein fibrillation: FRET studies of Y125W/Y133F/Y136F alpha-synuclein. J. Mol. Biol. 353, 357-372. doi: 10.1016/j.jmb.2005.08.046

Kiely, A. P., Asi, Y. T., Kara, E., Limousin, P., Ling, H., Lewis, P., et al. (2013). $\alpha$-Synucleinopathy associated with G51D SNCA mutation: a link between
Parkinson's disease and multiple system atrophy? Acta Neuropathol. 125, 753-769.

Kim, D. H., Park, S. J., Kim, J. M., Jeon, S. J., Cho, Y. W., Son, K. H., et al. (2011). Cognitive dysfunctions induced by a cholinergic blockade and A $\beta$ 25-35 peptide are attenuated by salvianolic acid B. Neuropharmacology 61, 1432-1440. doi: 10.1016/j.neuropharm.2011.08.038

Kim, Y. J. (2007). Antimelanogenic and antioxidant properties of gallic acid. Biol. Pharm. Bull. 30, 1052-1055. doi: 10.1248/bpb.30.1052

Kreis, W., Kaplan, M. H., Freeman, J., Sun, D. K., and Sarin, P. S. (1990). Inhibition of HIV replication by Hyssop officinalis extracts. Antiviral Res. 14, 323-337. doi: 10.1016/0166-3542(90)90051-8

Kroes, B. H., Van Den Berg, A. J., Quarles Van Ufford, H. C., Van Dijk, H., and Labadie, R. P. (1992). Anti-inflammatory activity of gallic acid. Planta Med. 58, 499-504. doi: 10.1055/s-2006-961535

Krüger, R., Kuhn, W., Müller, T., Woitalla, D., Graeber, M., Kösel, S., et al. (1998). Ala30Pro mutation in the gene encoding alpha-synuclein in Parkinson's disease. Nat. Genet. 18, 106-108. doi: 10.1038/ng0298-106

Leuner, K., Hauptmann, S., Abdel-Kader, R., Scherping, I., Keil, U., Strosznajder, J. B., et al. (2007). Mitochondrial dysfunction: the first domino in brain aging and Alzheimer's disease? Antioxid Redox Signal. 9, 1659-1675. doi: 10.1089/ars. 2007.1763

Levine, H., Lampe, L., Abdelmoti, L., and Augelli-Szafran, C. E. (2012). Dihydroxybenzoic acid isomers differentially dissociate soluble biotinyl-A $\beta$ (1-42) oligomers. Biochemistry 51, 307-315. doi: 10.1021/bi201288x

Levy-Sakin, M., Shreberk, M., Daniel, Y., and Gazit, E. (2009). Targeting insulin amyloid assembly by small aromatic molecules: toward rational design of aggregation inhibitors. Islets 1, 210-215. doi: 10.4161/isl.1.3.9609

Liu, K. C., Ho, H. C., Huang, A. C., Ji, B. C., Lin, H. Y., Chueh, F. S., et al. (2011). Gallic acid provokes DNA damage and suppresses DNA repair gene expression in human prostate cancer PC-3 cells. Environ. Toxicol. 28, 579-587. doi: 10.1002/ tox. 20752

Luk, K. C., Kehm, V., Carroll, J., Zhang, B., O'brien, P., Trojanowski, J. Q., et al. (2012). Pathological $\alpha$-synuclein transmission initiates Parkinson-like neurodegeneration in nontransgenic mice. Science 338, 949-953. doi: 10.1126/science. 1227157

Masliah, E., Rockenstein, E., Veinbergs, I., Mallory, M., Hashimoto, M., Takeda, A., et al. (2000). Dopaminergic loss and inclusion body formation in alphasynuclein mice: implications for neurodegenerative disorders. Science 287, 1265-1269. doi: 10.1126/science.287.5456.1265

Masuda, M., Suzuki, N., Taniguchi, S., Oikawa, T., Nonaka, T., Iwatsubo, T., et al. (2006). Small molecule inhibitors of alpha-synuclein filament assembly. Biochemistry 45, 6085-6094. doi: 10.1021/bi0600749

Mosmann, T. (1983). Rapid colorimetric assay for cellular growth and survival: application to proliferation and cytotoxicity assays. J. Immunol. Methods 65, 55-63. doi: 10.1016/0022-1759(83)90303-4

Ono, K., and Yamada, M. (2006). Antioxidant compounds have potent antifibrillogenic and fibril-destabilizing effects for alpha-synuclein fibrils in vitro. J. Neurochem. 97, 105-115. doi: 10.1111/j.1471-4159.2006.03707.x

Perez, R. G., Waymire, J. C., Lin, E., Liu, J. J., Guo, F., and Zigmond, M. J. (2002). A role for alpha-synuclein in the regulation of dopamine biosynthesis. J. Neurosci. 22, 3090-3099.

Perier, C., and Vila, M. (2012). Mitochondrial biology and Parkinson's disease. Cold Spring Harb. Perspect. Med. 2:a009332. doi: 10.1101/cshperspect.a009332

Polymeropoulos, M. H., Lavedan, C., Leroy, E., Ide, S. E., Dehejia, A., Dutra, A., et al. (1997). Mutation in the alpha-synuclein gene identified in families with Parkinson's disease. Science 276, 2045-2047. doi: 10.1126/science.276.53 21.2045

Porat, Y., Abramowitz, A., and Gazit, E. (2006). Inhibition of amyloid fibril formation by polyphenols: structural similarity and aromatic interactions as a common inhibition mechanism. Chem. Biol. Drug. Des. 67, 27-37. doi: 10.1111/j.1747-0285.2005.00318.x

Reinke, A. A., and Gestwicki, J. E. (2007). Structure-activity relationships of amyloid beta-aggregation inhibitors based on curcumin: influence of linker length and flexibility. Chem. Biol. Drug. Des. 70, 206-215. doi: 10.1111/j.17470285.2007.00557.x

Sameri, M. J., Sarkaki, A., Farbood, Y., and Mansouri, S. M. (2011). Motor disorders and impaired electrical power of pallidal EEG improved by gallic acid in animal model of Parkinson's disease. Pak. J. Biol. Sci. 14, 1109-1116. doi: 10.3923/pjbs.2011.1109.1116 
Schmitt, K., Grimm, A., Kazmierczak, A., Strosznajder, J. B., Götz, J., and Eckert, A. (2012). Insights into mitochondrial dysfunction: aging, amyloid- $\beta$, and tauA deleterious trio. Antioxid Redox Signal 16, 1456-1466. doi: 10.1089/ars.2011. 4400

Seidler, A., Hellenbrand, W., Robra, B. P., Vieregge, P., Nischan, P., Joerg, J., et al. (1996). Possible environmental, occupational, and other etiologic factors for Parkinson's disease: a case-control study in Germany. Neurology 46, 1275-1284. doi: 10.1212/WNL.46.5.1275

Serpell, L. C., Berriman, J., Jakes, R., Goedert, M., and Crowther, R. A. (2000). Fiber diffraction of synthetic alpha-synuclein filaments shows amyloid-like cross-beta conformation. Proc. Natl. Acad. Sci. U.S.A. 97, 4897-4902. doi: 10.1073/pnas.97. 9.4897

Singleton, A. B., Farrer, M., Johnson, J., Singleton, A., Hague, S., Kachergus, J., et al. (2003). alpha-Synuclein locus triplication causes Parkinson's disease. Science 302, 841. doi: 10.1126/science. 1090278

Spillantini, M. G., and Goedert, M. (2000). The alpha-synucleinopathies: Parkinson's disease, dementia with Lewy bodies, and multiple system atrophy. Ann. N.Y. Acad. Sci. 920, 16-27. doi: 10.1111/j.1749-6632.2000.tb06900.x

Spillantini, M. G., Schmidt, M. L., Lee, V. M., Trojanowski, J. Q., Jakes, R., and Goedert, M. (1997). Alpha-synuclein in Lewy bodies. Nature 388, 839-840. doi: $10.1038 / 42166$

Stefanis, L. (2012). $\alpha$-Synuclein in Parkinson's disease. Cold Spring Harb. Perspect. Med. 2:a009399. doi: 10.1101/cshperspect.a009399

Taniguchi, S., Suzuki, N., Masuda, M., Hisanaga, S., Iwatsubo, T., Goedert, M., et al. (2005). Inhibition of heparin-induced tau filament formation byphenothiazines, polyphenols, and porphyrins. J. Biol. Chem. 280, 7614-7623. doi: 10.1074/jbc.M408714200

Taylor, J. P., Hardy, J., and Fischbeck, K. H. (2002). Toxic proteins in neurodegenerative disease. Science 296, 1991-1995. doi: 10.1126/science. 1067122

Trojanowski, J. Q., and Lee, V. M. (2003). Parkinson's disease and related alphasynucleinopathies are brain amyloidoses. Ann. N.Y. Acad. Sci. 991, 107-110. doi: 10.1111/j.1749-6632.2003.tb07468.x

Uversky, V. N. (2008). Alpha-synuclein misfolding and neurodegenerative diseases. Curr. Protein Pept. Sci. 9, 507-540. doi: 10.2174/138920308785915218

Vekrellis, K., Xilouri, M., Emmanouilidou, E., Rideout, H. J., and Stefanis, L. (2011). Pathological roles of $\alpha$-synuclein in neurological disorders. Lancet Neurol. 10, 1015-1025. doi: 10.1016/S1474-4422(11)70213-7

Veldman, B. A., Wijn, A. M., Knoers, N., Praamstra, P., and Horstink, M. W. (1998). Genetic and environmental risk factors in Parkinson's disease. Clin. Neurol. Neurosurg. 100, 15-26. doi: 10.1016/S0303-8467(98)00009-2

Vendruscolo, M., Paci, E., Dobson, C. M., and Karplus, M. (2001). Three key residues form a critical contact network in a protein folding transition state. Nature 409, 641-645. doi: 10.1038/35054591

Volpicelli-Daley, L. A., Luk, K. C., Patel, T. P., Tanik, S. A., Riddle, D. M., Stieber, A., et al. (2011). Exogenous $\alpha$-synuclein fibrils induce Lewy body pathology leading to synaptic dysfunction and neuron death. Neuron 72, 57-71. doi: 10.1016/j. neuron.2011.08.033

Wagner, J., Ryazanov, S., Leonov, A., Levin, J., Shi, S., Schmidt, F., et al. (2013). Anle138b: a novel oligomer modulator for disease-modifying therapy of neurodegenerative diseases such as prion and Parkinson's disease. Acta Neuropathol. 125, 795-813. doi: 10.1007/s00401-013-1114-9

Walsh, D. M., Klyubin, I., Fadeeva, J. V., Cullen, W. K., Anwyl, R., Wolfe, M. S., et al. (2002). Naturally secreted oligomers of amyloid beta protein potently inhibit hippocampal long-term potentiation in vivo. Nature 416, 535-539. doi: 10.1038/ $416535 \mathrm{a}$

Wood, N. (1997). Genes and parkinsonism. J. Neurol. Neurosurg. Psychiatry 62, 305-309. doi: 10.1136/jnnp.62.4.305

Yang, C. S., Chung, J. Y., Yang, G. Y., Li, C., Meng, X., and Lee, M. J. (2000). Mechanisms of inhibition of carcinogenesis by tea. Biofactors 13, 73-79. doi: 10.1002/biof.5520130113

Yang, F., Lim, G. P., Begum, A. N., Ubeda, O. J., Simmons, M. R., Ambegaokar, S. S., et al. (2005). Curcumin inhibits formation of amyloid beta oligomers and fibrils, binds plaques, and reduces amyloid in vivo. J. Biol. Chem. 280, 5892-5901. doi: 10.1074/jbc.M404751200

Zarranz, J. J., Alegre, J., Gómez-Esteban, J. C., Lezcano, E., Ros, R., Ampuero, I., et al. (2004). The new mutation, E46K, of alpha-synuclein causes Parkinson and Lewy body dementia. Ann. Neurol. 55, 164-173. doi: 10.1002/ana.10795

Zhu, M., Rajamani, S., Kaylor, J., Han, S., Zhou, F., and Fink, A. L. (2004). The flavonoid baicalein inhibits fibrillation of alpha-synuclein and disaggregates existing fibrils. J. Biol. Chem. 279, 26846-26857. doi: 10.1074/jbc.M4031 29200

Conflict of Interest Statement: The authors declare that the research was conducted in the absence of any commercial or financial relationships that could be construed as a potential conflict of interest.

Received: 10 April 2014; paper pending published: 27 May 2014; accepted: 15 July 2014; published online: 05 August 2014.

Citation: Ardah MT, Paleologou KE, Lv G, Abul Khair SB, Kazim AS, Minhas ST, AlTel TH, Al-Hayani AA, Haque ME, Eliezer D and El-Agnaf OMA (2014) Structure activity relationship of phenolic acid inhibitors of $\alpha$-synuclein fibril formation and toxicity. Front. Aging Neurosci. 6:197. doi: 10.3389/fnagi.2014.00197

This article was submitted to the journal Frontiers in Aging Neuroscience.

Copyright (C) 2014 Ardah, Paleologou, Lv, Abul Khair, Kazim, Minhas, Al-Tel, AlHayani, Haque, Eliezer and El-Agnaf. This is an open-access article distributed under the terms of the Creative Commons Attribution License (CC BY). The use, distribution or reproduction in other forums is permitted, provided the original author(s) or licensor are credited and that the original publication in this journal is cited, in accordance with accepted academic practice. No use, distribution or reproduction is permitted which does not comply with these terms. 